THE ASTROPHYSICAL JoURNAL, 416:769-786, 1993 October 20

(C) 1993. The American Astronomical Society. All rights reserved. Printed in U.S.A.

\title{
CIRCUMSTELLAR PROPERTIES OF S STARS. I. DUST FEATURES
}

\author{
P. S. CHEN ${ }^{1}$ AND SUN KWOK \\ Department of Physics and Astronomy, University of Calgary, 2500 University Drive, NW, Calgary, Alberta, Canada T2N 1N4
}

Received 1992 November 30; accepted 1993 March 31

\begin{abstract}
The 1435 stars currently classified as of $\mathrm{S}$ spectral type have been searched for association with the IRAS Point Source Catalog. Among the $835 \mathrm{~S}$ stars with IRAS counterparts, 149 are found to have usable IRAS low-resolution spectra. The circumstellar properties of these $S$ stars are classified using the LRS. We find that the circumstellar properties of $S$ stars are very similar to those of $M$ stars. For the $S$ stars that have LRS, silicate emission objects form the largest group. Only four objects of the whole sample show the $\mathrm{SiC}$ emission feature commonly found in carbon stars. Some of the $\mathbf{S}$ stars that show a photospheric continuum with no infrared excess may not be thermal pulsing AGB stars but are mass transfer binaries.

We propose a new classification scheme for AGB stars, taking into account both the photospheric and circumstellar spectral properties. This scheme recognizes the importance of mass loss on the evolution of AGB stars. The evolutionary status of $S$ stars is discussed in terms of their circumstellar properties, and an evolutionary scenario is proposed to reconcile the visible photospheric properties and the circumstellar properties of S stars.
\end{abstract}

Subject headings: circumstellar matter - dust, extinction - stars: AGB and post-AGB

\section{INTRODUCTION}

Stars on the asymptotic giant branch are generally classified into oxygen- $(\mathrm{M})$ or carbon-rich $(\mathrm{C})$ based on photospheric abundances. Since the discovery of circumstellar dust emission in the early 1970s (Woolf \& Ney 1969; Treffers \& Cohen 1973), there has been a good one-to-one correspondence between the chemical makeup of circumstellar dust and photospheric spectral types. The 9.7 and $18 \mu \mathrm{m}$ silicate features are associated with $\mathrm{M}$ stars, whereas the $11.3 \mu \mathrm{m} \mathrm{SiC}$ feature is found in $\mathrm{C}$ stars. However, the circumstellar properties of another major spectral type - S stars - has not been systematically studied.

$\mathrm{S}$ stars are peculiar red giant stars with enriched $s$-process elements and have the ratio of oxygen to carbon abundance that are intermediate between $\mathbf{M}$ and $\mathrm{C}$ stars (Smith \& Lambert 1985, 1986; Lambert et al. 1986). Since the [C/O] ratio is approximately unity, almost all oxygen and carbon atoms in the atmosphere of $\mathrm{S}$ stars are consumed in the $\mathrm{CO}$ molecule, and the molecules that are formed in S star atmospheres can be quite different from those in $M$ and $C$ stars. Specifically, $\mathrm{S}$ stars are characterized by the prominence of the $\mathrm{ZrO}$ band over the $\mathrm{TiO}$ band. Consequently, $\mathrm{S}$ stars are often suggested as objects in transition between $\mathrm{M}$ and $\mathrm{C}$ stars.

However, the traditional interpretation of the M-S-C sequence as an evolutionary sequence has been challenged by recent studies. Jorissen \& Mayor (1988) found that some nonvariable $\mathrm{S}$ stars without Tc may be binaries (Jorissen \& Mayor 1988). On the other hand, carbon stars with circumstellar silicate features have been suggested as transition objects between M and C stars (Willems \& de Jong 1988; Chan \& Kwok 1988, 1991), and the circumstellar properties of $S$ stars cannot be readily fitted into this picture (Chan \& Kwok 1990).

In the mid-infrared, both $\mathrm{M}$ and $\mathrm{C}$ stars have well-defined colors and form homogeneous groups in the IRAS color-color diagram (Volk \& Kwok 1988; van der Veen \& Habing 1988;

\footnotetext{
${ }^{1}$ On leave from Yunan Observatory, Academic Sinica, People's Republic of China.
}

Walker \& Cohen 1988). However, S stars have no unique colors in the near-infrared (Chen et al. 1988; Noguchi et al. $1991)$ or in the mid-infrared. In the $I R A S$ color-color diagram, the distribution of $\mathbf{S}$ stars overlaps extensively with those of $\mathbf{M}$ and $\mathrm{C}$ stars and does not form a well-defined group by itself.

The recent publication of $S$ star catalogs and the availability of photometric and spectroscopic data from the IRAS sky survey have made possible a comprehensive study of the circumstellar properties of S stars. For the first time, it is possible to obtain answers to the following questions: What fraction of $\mathrm{S}$ stars have infrared excess indicative of mass loss? What is the composition of the circumstellar dust of S stars? Are there any correlations between the photospheric spectral types and the circumstellar spectral types? If the circumstellar dust of $\mathbf{M}$ and $\mathrm{C}$ stars are indicators of the mass-loss history and evolutionary status of these stars, can any definite statement by made on the nature of S stars?

In this paper, we have assembled a sample of $S$ stars that have associations with the IRAS Point Source Catalog. The $I R A S$ low-resolution spectra of these stars are examined and the nature of the circumstellar dust classified. The distributions of these subgroups of $\mathrm{S}$ stars in the IRAS color-color diagram are interpreted with an evolutionary model.

\section{IRAS LRS SPECTRA OF S STARS}

The first comprehensive catalog of S stars is the General Catalogue of S stars (Stephenson 1976, hereafter GCSS) which contains 741 objects. In a later revised version, General Catalogue of Galactic S Stars, Second Edition (Stephenson 1984, hereafter GCGSS), the number of S stars has increased to 1347. A number of $\mathbf{M}$ or $\mathbf{C}$ stars previously misclassified as $\mathbf{S}$ stars in GCSS are also eliminated in GCGSS. Furthermore, Table 2 of the General Catalog of Cool Galactic Carbon Stars, Second Edition (Stephenson 1989, hereafter GCCGCS) lists a number of S (or MS and SC) stars which have previously been misclassified as carbon stars. An additional $75 \mathrm{~S}$ stars are reported by Stephenson (1990) since the publication of GCGSS. Com- 
bining the entries in these catalogs, we have a total of $1435 \mathrm{~S}$ stars known to date.

The IRAS Point Source Catalog (1988, hereafter PSC) lists 483 associations with GCSS; four (GCSS 447, 539, 549, and 584) are now known to have been misclassified and are not $S$ stars. For the remaining $694 \mathrm{~S}$ stars not in the GCSS, we have searched for IRAS associations with the following procedure: (1) first we search for PSC sources within a $90^{\prime \prime}$ radius (the same search radius as employed in the PSC for catalog associations in the GCSS) of the S star position; (2) the position error ellipses of the IRAS sources together with the S star optical position are plotted on the atlas of the HST guide star catalog (1989); (3) the GCGSS $b$ or $v$ magnitudes are compared with the HST guide star magnitudes to identify the IRAS counterpart to the $S$ star. Using this procedure, we found 355 new associations, together with the previous 479 giving a total of $834 \mathrm{~S}$ stars with IRAS associations.

We note that the above procedure is more rigorous than the procedure used in the PSC, which basically does not go beyond step (1). As an example GCGSS 973 is within $90^{\prime \prime}$ of IRAS $17180-3655$ but is located more than 3 times the error radius from the IRAS source. We have therefore rejected the association. A total of eight objects were rejected this way. Since the size of our search sample is similar to that of the GCSS, one may expect a similar number of problem cases in the PSC associations.

We have also examined the $I R A S$ low-resolution spectra (LRS) of this sample by using the following references: Atlas of Low-Resolution IRAS Spectra (1986, hereafter LRS Atlas), Volk \& Cohen (1989), and Volk et al. (1991). For sources with $12 \mu \mathrm{m}$ fluxes between 7 and $20 \mathrm{Jy}$, LRS spectra were extracted at the IRAS Data Analysis Facility at the University of Calgary. Out of 834 sources in the sample, 149 sources have LRS spectra available. These include $121 \mathrm{~S}$ stars, 19 MS stars, and 9 SC stars.

In Table 1 we list the $\mathrm{S}$ stars with IRAS LRS. The sources are grouped by the University of Calgary LRS classifications using the letter codes defined by Volk \& Cohen (1989) and ordered by R.A. within each group. Among the $121 \mathrm{~S}$ stars, 39 are in group $\mathrm{E}$ (showing the $9.7 \mu \mathrm{m}$ silicate feature in emission), 38 in group $\mathrm{F}$ (featureless dust continuum), 29 in group $\mathrm{S}$ (stellar photospheric continuum), 3 in group $\mathrm{C}(11.3 \mu \mathrm{m} \mathrm{SiC}$ emission), 2 in group $U$ (unusual spectrum), and 10 in group I (noisy or incomplete spectrum). Also listed in Table 1 are the $I R A S$, GCSS, GCGSS, and variable star (Kholopov 1985, hereafter GCVS) names. For sources without GCSS numbers, the IRAS associations are indentified in this paper. The visual spectral type given in GCGSS are listed in column (6) with the same notation as used in GCGSS. Column (7) gives the LRS classification codes from the LRS Atlas (two-digit numbers). Columns (8) and (9) list, respectively, the probability of variability and the IRAS flux quality as given in the PSC. The variability type and the period from the GCVS are given in columns (10) and (11), respectively. Finally, column (12) is the absolute value of the galactic latitude for the sample.

In Table 2 we list the 19 MS stars and nine SC stars with LRS spectra. All columns in Table 2 have the same meaning as in Table 1.

The LRS spectra of the sources in Tables 1 and 2 are plotted in Figure 1 and Figure 2, respectively.

\section{CIRCUMSTELLAR FEATURES OF S STARS}

From the classification of the circumstellar dust of S stars, we see that silicate emission (E) form the largest group (32\%), followed by featureless dust continuum (31\%), stellar continuum $(24 \%)$, and only a small fraction shows $\mathrm{SiC}$ emission (2\%). From Table 2, 37\% of MS stars have silicate emission features, whereas only $10 \%$ of SC stars have $11.3 \mu \mathrm{m} \mathrm{SiC}$ features. The relatively few number of $\mathrm{SiC}-\mathrm{S}$ stars is not entirely surprising because the $[\mathrm{C} / \mathrm{O}]$ abundance ratio is less than unity for most sources in Table 1 . The $[\mathrm{C} / \mathrm{O}]$ abundance ratio is $\sim 0.6$ for $\mathrm{MS}$ stars, 0.8 for $S$ stars (Smith \& Lambert 1985, 1986), and 0.98-1.0 for SC stars with spectral type earlier than SCx/8 (Keenan \& Boeshaar 1980).

Since many $\mathbf{S}$ stars were cataloged as a result of lowresolution surveys, the possibility of misclassification is real. For example, eight of the group E and one of the group U S stars have been found by Lloyd Evans \& Catchpole (1989) to be $\mathbf{M}$ stars. A more precise photospheric classification is needed before definite conclusions on the circumstellar properties of S stars can be drawn.

\subsection{Mira Variables}

Among sources with known variability types, Mira variables constitute $79 \%$ of group E, $50 \%$ of group F, and $24 \%$ of group $\mathrm{S}$. This is consistent with the general expectation that Mira variables are losing mass. Average periods of Mira variables are 425 days for group E, 365 days for group F, and 335 days for group S. Since the pulsation periods and mass-loss rates are expected to increase as the star ascend the AGB, it is reasonable that the S-type Mira variables that show the most definite mass loss (group E) also have the longest periods.

For stars that have PSC probability of the variability greater than $75 \%, 14(38 \%)$ and in group E, $7(18 \%)$ in group F, and 4 $(10 \%)$ in group S. Again, there seems to be a correlation between pulsation and infrared excess.

It is worth noting that no $\mathrm{S}$ stars show the silicate feature in absorption. This suggests that they all have relatively thin circumstellar shells and have mass-loss rates much lower than those of late $\mathrm{M}$ (e.g., OH/IR) stars.

While most Mira variables show infrared excesses and evidence of mass loss, five S-type Miras (U Cas, T Cam, V865 Aql, FF Cyg, and GR Cyg), and one SC Mira (R CMi) are classified as group $S$. The fact that their mid-infrared spectra are consistent with the Rayleigh-Jeans tail of their stellar photospheric continua suggests that they are not undergoing significant mass loss.

\subsection{Peculiar Features}

It is interesting to note that some LRS spectra in Figure 1 have abnormal shapes. For example, IRAS $11169-6111$ is classified as having silicate emission, but its emission peak is at $\sim 13.2 \mu \mathrm{m}$, and its continuum continues to rise beyond $15 \mu \mathrm{m}$. Among the group $\mathrm{E}$ objects there are a number of sources (sources $8,11,13,14,15,16,20,21,22,27,35$, and 36) that show a broad emission feature around 9-13 $\mu \mathrm{m}$. ${ }^{2}$ There are also some group $F$ sources that show broad emissions in the same region, although the features are not as strong as the above objects. Little-Marenin \& Little (1988) suggest that such broad features can be explained by a mixture of the $10 \mu \mathrm{m}$ silicate feature and the $11.3 \mu \mathrm{m} \mathrm{SiC}$ feature.

The one group U source (source $110=$ IRAS 16488-4407) displays unknown emission features around $8 \mu \mathrm{m}$. This feature is also known to exist in some carbon stars (Volk 1993).

One MS star (source $139=$ V535 Ori) has a broad emission feature around 9.4-12 $\mu \mathrm{m}$ (Fig. 2). The SC star IRAS

${ }^{2}$ Sources 11 and 20 were classified as having the SiC feature by Skinner, Griffin, \& Whitmore (1990). 
TABLE 1

S-TYPE STARS IN DIFFERENT LRS GROUPS

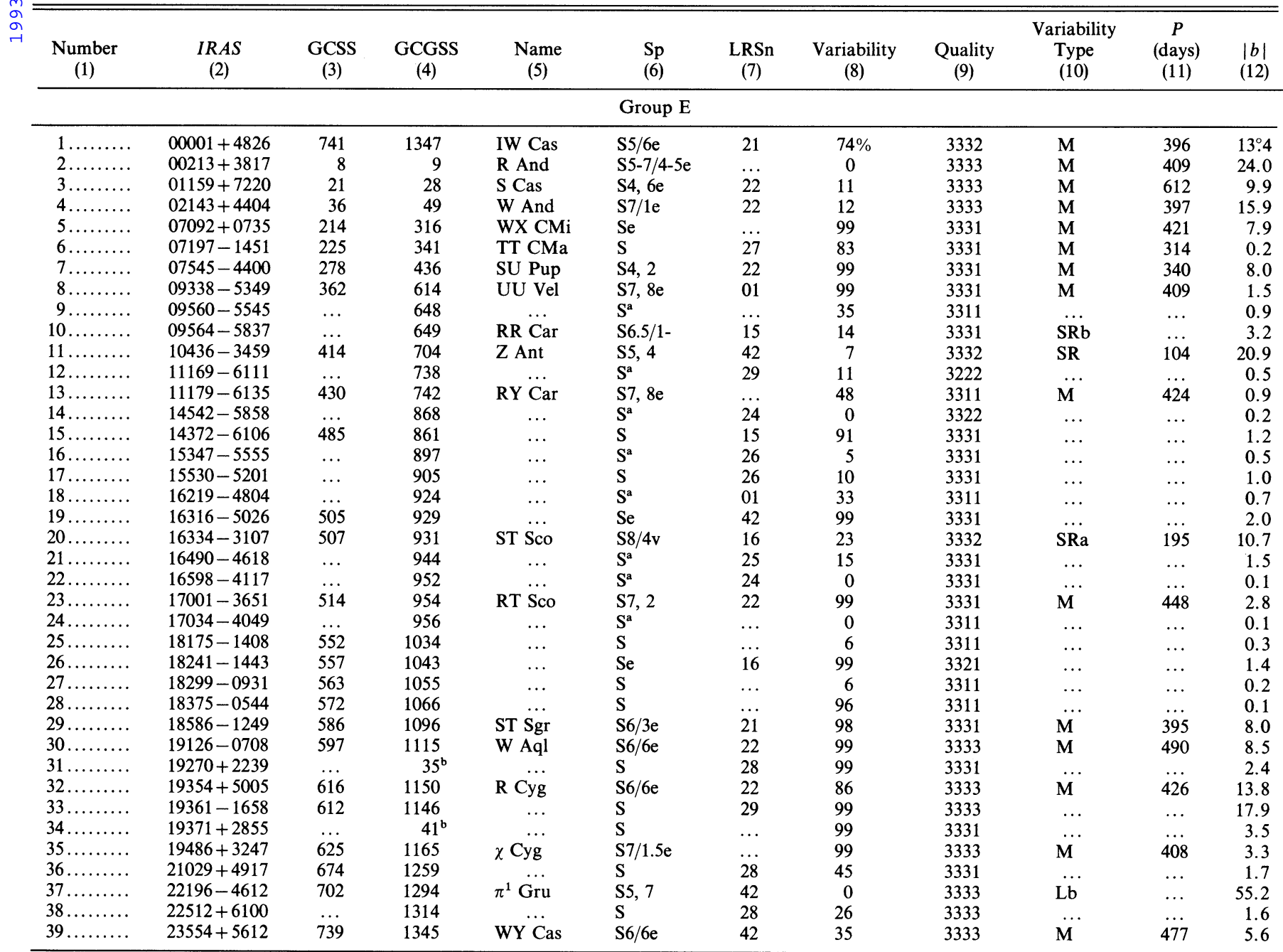

\begin{tabular}{|c|c|c|c|c|c|c|c|c|c|c|c|}
\hline & & & & & Group F & & & & & & \\
\hline $40 \ldots \ldots \ldots$ & $00135+4644$ & 5 & 6 & $X$ And & $\mathrm{S} 4 / 7 \mathrm{e}$ & 16 & 47 & 3332 & $\mathbf{M}$ & 346 & 15.4 \\
\hline $41 \ldots \ldots \ldots$ & $00445+3224$ & 13 & 14 & RW And & $\mathrm{S} 6 / 2 \mathrm{e}$ & 22 & 74 & 3332 & $\mathbf{M}$ & 429 & 30.2 \\
\hline $42 \ldots \ldots \ldots$ & $00578+5620$ & 17 & 20 & V365 Cas & S6/3 & 16 & 19 & 3331 & $\mathrm{SRb}$ & $\ldots$ & 6.2 \\
\hline $43 \ldots \ldots \ldots$ & $01113+2815$ & 20 & 26 & & $\mathrm{~S} 3 / 2$ & & 0 & 3321 & & $\ldots$ & 34.1 \\
\hline $44 \ldots \ldots \ldots$ & $03452+5301$ & 62 & 82 & WX Cam & $\mathrm{S} 5 / 6$ & 01 & 25 & 3331 & $\mathrm{Lb}$ & & 0.9 \\
\hline $45 \ldots \ldots \ldots$ & $04204+4149$ & 68 & 92 & GS Per & $\mathrm{Sp}$ & $\ldots$ & 99 & 3331 & $\mathbf{M}$ & 230 & 5.3 \\
\hline $46 \ldots \ldots \ldots$ & $04430-2356$ & & 109 & & $\mathrm{~S}$ & 16 & 11 & 3331 & & & 1.8 \\
\hline $47 \ldots \ldots \ldots$ & $04543+4829$ & 86 & 116 & TV Aur & S5/6 & & 0 & 3331 & $\mathrm{SRb}$ & 182 & 16.5 \\
\hline $48 \ldots \ldots \ldots$ & $05429-0415$ & $\ldots$ & 159 & & $\mathrm{~S} 5 * 3$ & $\dddot{16}$ & 2 & 3321 & $\ldots$ & $\ldots$ & 16.6 \\
\hline $49 \ldots \ldots \ldots$ & $05495+1547$ & $\ldots$ & 168 & Z Tau & $\mathrm{S} 7.5,1 \mathrm{e}$ & $\ldots$ & 87 & 3321 & $\dddot{M}$ & $\ddot{466}$ & 5.4 \\
\hline $50 \ldots \ldots \ldots$ & $06266-1148$ & $\cdots$ & 221 & & $\mathrm{~S}-* 2 \mathrm{e}$ & & 99 & 3331 & & & 10.3 \\
\hline $51 \ldots \ldots \ldots$ & $06331+1415$ & 156 & 231 & DY $\dddot{G e m}$ & S8, 5 & 42 & 23 & 3333 & $\dddot{\mathrm{SRa}}$ & 115 & 3.1 \\
\hline $52 \ldots \ldots \ldots$ & $06571+5524$ & 197 & 283 & R Lyn & $\mathrm{S} 5 / 5 \mathrm{e}$ & 16 & 99 & 3331 & M & 379 & 23.4 \\
\hline $53 \ldots \ldots \ldots$ & $07043+2246$ & 206 & 307 & R Gem & $\mathrm{S} 5 / 5$ & 16 & 99 & 3333 & $\mathbf{M}$ & 370 & 13.5 \\
\hline $54 \ldots \ldots \ldots$ & $07461-3705$ & & 408 & $\ldots$ & $\mathrm{S} 6 * 1$ & 14 & 16 & 3331 & $\ldots$ & $\ldots$ & 5.9 \\
\hline $55 \ldots \ldots \ldots$ & $08403-3853$ & 327 & 542 & $\cdots$ & S4, 6 & 16 & 12 & 3331 & $\ldots$ & $\ldots$ & 1.9 \\
\hline $56 \ldots \ldots \ldots$ & $12337-6124$ & 448 & 799 & $\ldots$ & $\mathrm{S}$ & $\ldots$ & 2 & 3321 & & & 1.1 \\
\hline $57 \ldots \ldots \ldots$ & $13240-5742$ & & 821 & EE Cen & $56 * 3$ & 14 & 14 & 3332 & SR & 198 & 4.6 \\
\hline $58 \ldots \ldots \ldots$ & $14510-6052$ & 488 & 867 & & S6, 2 & $\ldots$ & 43 & 3311 & & & 1.7 \\
\hline $59 \ldots \ldots \ldots$ & $15030-4116$ & 491 & 872 & GI Lup & S7, 8e & $\ldots$ & 33 & 3331 & $\dddot{M}$ & $\dddot{326}$ & 14.7 \\
\hline $60 \ldots \ldots \ldots$ & $16097-6158$ & 501 & 914 & Y Tra & $\mathrm{S} 4,1$ & $\cdots$ & 49 & 3331 & $\mathbf{M}$ & 323 & 8.0 \\
\hline $61 \ldots \ldots \ldots$ & $16545-3230$ & $\ldots$ & 949 & EW Sco & $\mathrm{S} 5 * 2$ & $\ldots$ & 0 & 3321 & $\mathrm{Lb}$ & $\ldots$ & 6.4 \\
\hline $62 \ldots \ldots \ldots$ & $16552-5335$ & 513 & 948 & $\ldots$ & $\mathrm{S} 4,4$ & $\ldots$ & 53 & 3321 & $\ldots$ & $\ldots$ & 6.8 \\
\hline $63 \ldots \ldots \ldots$ & $17186-2914$ & 523 & 975 & & $\mathrm{~S}$ & 15 & 99 & 3321 & $\ldots$ & $\ldots$ & 4.2 \\
\hline $64 \ldots \ldots \ldots$ & $17188-1433$ & 524 & 976 & FT Ser & S5, 8 & & 10 & 3321 & $\dddot{\mathrm{Lb}}$ & $\cdots$ & 12.4 \\
\hline $65 \ldots \ldots \ldots$ & $17478-2957$ & $\ldots$ & 1001 & V762 Sgr & $56 * 4$ & $\dddot{14}$ & 8 & 2321 & $\mathbf{M}$ & $\dddot{444}$ & 1.5 \\
\hline
\end{tabular}


TABLE 1-Continued

\begin{tabular}{|c|c|c|c|c|c|c|c|c|c|c|c|}
\hline $\begin{array}{c}\text { Number } \\
\text { (1) }\end{array}$ & $\begin{array}{l}I R A S \\
\quad(2)\end{array}$ & $\begin{array}{c}\text { GCSS } \\
\text { (3) }\end{array}$ & $\begin{array}{c}\text { GCGSS } \\
(4)\end{array}$ & $\underset{(5)}{\text { Name }}$ & $\begin{array}{l}\mathrm{Sp} \\
(6)\end{array}$ & $\begin{array}{c}\text { LRSn } \\
\text { (7) }\end{array}$ & $\begin{array}{c}\text { Variability } \\
\text { (8) }\end{array}$ & $\begin{array}{l}\text { Quality } \\
\text { (9) }\end{array}$ & $\begin{array}{c}\text { Variability } \\
\text { Type } \\
\text { (10) }\end{array}$ & $\begin{array}{c}P \\
\text { (days) } \\
(11)\end{array}$ & $\begin{array}{l}|b| \\
(12)\end{array}$ \\
\hline \multicolumn{12}{|c|}{ Group $\mathrm{F}-$ Continued } \\
\hline ..... & $17521-2907$ & 535 & 1007 & V745 Sgr & $\mathrm{Se}$ & 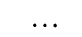 & 99 & 3321 & $\mathbf{M}$ & 380 & 1.9 \\
\hline $67 \ldots \ldots \ldots$ & $17562-1133$ & 538 & 1011 & $\ldots$ & $\mathbf{S}$ & 01 & 56 & 3331 & $\ldots$ & $\ldots$ & 6.1 \\
\hline $68 \ldots \ldots \ldots$ & $17594-2451$ & $\ldots$ & 1018 & $\ldots$ & $\mathrm{S}-* 2$ & $\ldots$ & 20 & 3311 & $\ldots$ & $\ldots$ & 1.2 \\
\hline $69 \ldots \ldots \ldots$ & $18284-0924$ & 560 & 1052 & BP Sct & S5/5 & $\ldots$ & 2 & 3311 & $\mathbf{L b}$ & $\ldots$ & 0.2 \\
\hline $70 \ldots \ldots \ldots$ & $19133-1703$ & 598 & 1117 & T Sgr & $\mathrm{S} 5 / 6 \mathrm{e}$ & $\ldots$ & 58 & 3333 & $\mathbf{M}$ & 392 & 13.0 \\
\hline $71 \ldots \ldots \ldots$ & $19311+2332$ & 609 & 1141 & EP Vul & S6/5 & 16 & 36 & 3331 & $\mathbf{L b}$ & $\ldots$ & 2.0 \\
\hline $72 \ldots \ldots \ldots$ & $20044+2417$ & 634 & 1189 & DK Vul & $\mathrm{S} 4,2$ & 16 & 3 & 3333 & $\mathrm{SRa}$ & 370 & 4.1 \\
\hline $73 \ldots \ldots \ldots$ & $20120-4433$ & 637 & 1196 & RZ Sgr & S4, 4 & 16 & 29 & 3333 & $\mathrm{SRb}$ & 223 & 33.2 \\
\hline $74 \ldots \ldots \ldots$ & $20296+3223$ & 651 & 1224 & AD Cyg & S5/5 & $\ldots$ & 11 & 3311 & $\mathbf{L b}$ & $\ldots$ & 4.1 \\
\hline $75 \ldots \ldots \ldots$ & $22159-2109$ & 700 & 1292 & X Aqr & S6/3e & $\ldots$ & 12 & 3321 & $\mathbf{M}$ & 312 & 54.7 \\
\hline $76 \ldots \ldots \ldots$ & $22479+5923$ & 715 & 1310 & CV Cep & S6/2 & $\ldots$ & 18 & 3311 & SR & 60 & 0.3 \\
\hline $77 \ldots \ldots \ldots$ & $23595-1457$ & 740 & 1346 & W Cet & S5-7/ & 16 & 8 & 3331 & $\mathbf{M}$ & 351 & 73.1 \\
\hline \multicolumn{12}{|c|}{ Group S } \\
\hline$\ldots \ldots$ & $00435+4758$ & 11 & 12 & U Cas & $\mathrm{S} 5 / 3 \mathrm{e}$ & 01 & 27 & 3331 & $\mathbf{M}$ & 278 & 14.6 \\
\hline $79 \ldots \ldots \ldots$ & $02228+3753$ & 43 & 57 & BI And & $S 8 / 4 v$ & 01 & 4 & 3332 & SR & 195 & 21.1 \\
\hline $80 \ldots \ldots \ldots$ & $03377+6303$ & 60 & 79 & DB Cam & $\mathrm{S} 4 / 2$ & 18 & 23 & 3331 & Lb & $\ldots$ & 6.4 \\
\hline $81 \ldots \ldots \ldots$ & $04123+2357$ & $\ldots$ & 89 & $\ldots$ & $\mathrm{S}$ & $\ldots$ & 4 & 3331 & $\ldots$ & $\ldots$ & 19.0 \\
\hline $82 \ldots \ldots \ldots$ & $04352+6602$ & 75 & 103 & T Cam & S6/5e & 17 & 98 & 3333 & $\mathbf{M}$ & 374 & 12.8 \\
\hline $83 \ldots \ldots \ldots$ & $05199-0842$ & 98 & 133 & $\ldots$ & $\mathrm{S} 4,1$ & 16 & 2 & 3331 & $\ldots$ & $\ldots$ & 23.7 \\
\hline $84 \ldots \ldots \ldots$ & $06197+0327$ & 146 & 212 & FU Mon & $\mathrm{S} 7 / 7$ & 18 & 26 & 3332 & SR & 309 & 4.9 \\
\hline $85 \ldots \ldots \ldots$ & $07399-1045$ & 256 & 387 & SU Mon & S5/7 & 18 & 6 & 3331 & $\mathrm{SRb}$ & $\ldots$ & 6.1 \\
\hline $86 \ldots \ldots \ldots$ & $08098-2809$ & 296 & 474 & $\ldots$ & S4, 2 & $\ldots$ & 3 & 3321 & $\ldots$ & $\ldots$ & 3.1 \\
\hline $87 \ldots \ldots \ldots$ & $08214-3807$ & $\ldots$ & 500 & $\ldots$ & $S$ & 16 & 0 & 3321 & $\cdots$ & $\ldots$ & 0.6 \\
\hline $88 \ldots \ldots \ldots$ & $08348-3617$ & 320 & 533 & $\ldots$ & S5, 2 & 18 & 10 & 3331 & $\ldots$ & $\ldots$ & 2.7 \\
\hline $89 \ldots \ldots \ldots$ & $10389-5149$ & 409 & 696 & $\cdots$ & S5, 2 & 18 & 23 & 3331 & $\ldots$ & $\ldots$ & 5.8 \\
\hline $90 \ldots \ldots \ldots$ & $11098-3209$ & $\ldots$ & 729 & $\cdots$ & Swk & $\ldots$ & 6 & 3321 & $\ldots$ & $\ldots$ & 25.9 \\
\hline $91 \ldots \ldots \ldots$ & $12272-4127$ & $\ldots$ & 796 & $\ldots$ & Swk & 18 & 0 & 3331 & $\ldots$ & $\ldots$ & 21.0 \\
\hline $92 \ldots \ldots \ldots$ & $13079-8931$ & 453 & 804 & $\ldots$ & S5, 1 & 17 & 6 & 3331 & $\ldots$ & $\ldots$ & 26.9 \\
\hline $93 \ldots \ldots \ldots$ & $13372-7136$ & 468 & 826 & $\ldots$ & S6, 2 & 18 & 0 & 3331 & $\ldots$ & $\ldots$ & 9.4 \\
\hline $94 \ldots \ldots \ldots$ & $13477-6009$ & 473 & 834 & VX Cen & S8, 5 & 17 & 43 & 3331 & SR & 308 & 1.6 \\
\hline $95 \ldots \ldots \ldots$ & $16425-1902$ & $\ldots$ & 938 & $\ldots$ & Swk & 31 & 32 & 3331 & $\ldots$ & $\ldots$ & 16.8 \\
\hline $96 \ldots \ldots \ldots$ & $17188-4141$ & 522 & 974 & V635 Sco & S6...3+ & 17 & 3 & 3321 & Lb & $\ldots$ & 3.0 \\
\hline $97 \ldots \ldots \ldots$ & $17206-2826$ & 526 & 978 & V521 Oph & S5, 4 & 17 & 18 & 3331 & SRb & 320 & 4.3 \\
\hline $98 \ldots \ldots \ldots$ & $18300-1948$ & 562 & 1054 & V2003 Sgr & S5, 8 & $\ldots$ & 45 & 3311 & $\mathrm{SRb}$ & 380 & 5.0 \\
\hline $99 \ldots \ldots \ldots$ & $19008+1210$ & 588 & 1099 & V915 Aql & S5 ...2 & $\ldots$ & 80 & 3321 & $\mathrm{Lb}$ & $\ldots$ & 3.0 \\
\hline $100 \ldots \ldots \ldots$ & $20026+3640$ & 633 & 1188 & AA Cyg & S6...3 & 31 & 24 & 3331 & SRb & 213 & 2.9 \\
\hline $101 \ldots \ldots \ldots$ & $20100-6225$ & 636 & 1195 & $\therefore$ & S4, 4 & 18 & 0 & 3333 & $\ldots$ & $\ldots$ & 33.4 \\
\hline $102 \ldots \ldots \ldots$ & $20213+0047$ & 646 & 1211 & V865 Aql & S7, 2 & 17 & 46 & 3331 & $\mathbf{M}$ & 365 & 20.0 \\
\hline $103 \ldots \ldots \ldots$ & $20252+3623$ & 656 & 1219 & V441 Cyg & S4, 6 & 16 & 5 & 3311 & $\mathrm{SRa}$ & 375 & 10.5 \\
\hline $104 \ldots \ldots \ldots$ & $20369+3742$ & 655 & 1232 & FF Cyg & $\mathrm{S} 5 / 6 \mathrm{e}$ & $\ldots$ & 96 & 3311 & $\mathbf{M}$ & 323 & 2.1 \\
\hline $105 \ldots \ldots \ldots$ & $22476+4047$ & $\ldots$ & 1308 & RX Lac & $\mathrm{S} 7.5 / 1 \mathrm{e}$ & 16 & 14 & 3333 & $\mathrm{SRb}$ & 650 & 16.2 \\
\hline $106 \ldots \ldots \ldots$ & $22521+1640$ & 718 & 1315 & HR Peg & $\mathrm{S} 4 / 1$ & $\ldots$ & 0 & 3331 & $\mathrm{SRb}$ & 50 & 37.6 \\
\hline \multicolumn{12}{|c|}{ Group C } \\
\hline$\ldots \ldots$ & $13136-4426$ & 463 & 816 & UY Cen & S6/8 & 43 & 9 & 3333 & SR & 115 & 17.9 \\
\hline $108 \ldots \ldots \ldots$ & $15194-5115$ & ‥ & 886 & $\cdots$ & $S$ & 04 & 96 & 3332 & $\ldots$ & $\ldots$ & 4.7 \\
\hline $109 \ldots \ldots \ldots$ & $21027+3704$ & 673 & 1258 & GR Cyg & $\mathbf{S}$ & $\cdots$ & 99 & 3331 & $\mathbf{M}$ & $\cdots$ & 7.6 \\
\hline \multicolumn{12}{|c|}{ Group U } \\
\hline $110 \ldots \ldots \ldots$ & $16488-4407$ & $\cdots$ & 942 & $\cdots$ & $\mathbf{S}^{\mathbf{a}}$ & $\cdots$ & 3 & 3311 & $\cdots$ & $\cdots$ & 0.1 \\
\hline $111 \ldots \ldots \ldots$ & $18269-1111$ & $\cdots$ & 1047 & $\cdots$ & S-*3 & $\cdots$ & 25 & 3313 & $\cdots$ & $\cdots$ & 0.3 \\
\hline \multicolumn{12}{|c|}{ Group I } \\
\hline $112 \ldots \ldots \ldots$ & $07507-1129$ & 270 & 422 & NQ Pup & S5/2 & $\ldots$ & 38 & 3331 & Lb & & 8.0 \\
\hline $113 \ldots \ldots \ldots$ & $08188+1726$ & 305 & 494 & V Cnc & $\mathrm{S} 3 / 6 \mathrm{e}$ & $\ldots$ & 0 & 3311 & $\mathbf{M}$ & 272 & 27.5 \\
\hline $114 \ldots \ldots \ldots$ & $13226-6302$ & 465 & 820 & NZ Cen & $\mathbf{S}$ & $\ldots$ & 58 & 3311 & $\mathbf{M}$ & 382 & 0.7 \\
\hline $115 \ldots \ldots \ldots$ & $14500-4624$ & 487 & 866 & S Lup & $\mathrm{Se}$ & $\ldots$ & 39 & 3311 & $\mathbf{M}$ & 340 & 11.3 \\
\hline $116 \ldots \ldots \ldots$ & $16209-2808$ & 503 & 923 & $\ldots$ & $\mathbf{S}$ & $\ldots$ & 0 & 3331 & $\ldots$ & $\ldots$ & 14.7 \\
\hline $117 \ldots \ldots \ldots$ & $19451+0827$ & 621 & 1159 & QU Aql & $\mathrm{Se}$ & $\ldots$ & 79 & 3331 & $\mathbf{M}$ & 607 & 8.3 \\
\hline $118 \ldots \ldots \ldots$ & $19528-3827$ & $\ldots$ & 1170 & $\ldots$ & Swk & $\ldots$ & 1 & 3311 & $\ldots$ & $\ldots$ & 28.5 \\
\hline $119 \ldots \ldots \ldots$ & $20114+7702$ & 640 & 1200 & SZ Cep & $\mathrm{S} 5 / 6 \mathrm{e}$ & $\ldots$ & 97 & 3331 & $\mathbf{M}$ & 327 & 22.2 \\
\hline $120 \ldots \ldots \ldots$ & $21172-4819$ & 681 & 1268 & $\ldots$ & $\mathrm{S} 2,5$ & $\ldots$ & 95 & 3321 & $\ldots$ & $\ldots$ & 44.4 \\
\hline $121 \ldots \ldots \ldots$ & $23489+6235$ & 737 & 1342 & EO Cas & $\mathrm{Se}$ & $\ldots$ & 32 & 3321 & $\mathbf{M}$ & 445 & 0.8 \\
\hline
\end{tabular}

a Classified as M stars by Lloyd Evans \& Catchpole 1989.

b Star numbers from Stephenson 1990. 
TABLE 2

MS AND SC STARS WITH DIFFERENT LRS INDICES

\begin{tabular}{|c|c|c|c|c|c|c|c|c|c|c|c|c|}
\hline $\begin{array}{c}\text { Number } \\
\text { (1) }\end{array}$ & $\begin{array}{c}I R A S \\
\quad(2)\end{array}$ & $\begin{array}{c}\text { GCSS } \\
\text { (3) }\end{array}$ & $\begin{array}{c}\text { GCGSS } \\
\text { (4) }\end{array}$ & $\begin{array}{c}\text { Name } \\
(5)\end{array}$ & $\begin{array}{l}\text { Sp. } \\
(6)\end{array}$ & $\begin{array}{c}\text { LRSn } \\
(7)\end{array}$ & $\begin{array}{c}\text { LRS } \\
(8)\end{array}$ & $\begin{array}{c}\text { Variability } \\
\text { (9) }\end{array}$ & $\begin{array}{l}\text { Quality } \\
\text { (10) }\end{array}$ & $\begin{array}{c}\text { Variability } \\
\text { Type } \\
\text { (11) }\end{array}$ & $\begin{array}{c}P \\
\text { (days) } \\
(12)\end{array}$ & $\begin{array}{l}|b| \\
(13)\end{array}$ \\
\hline \multicolumn{13}{|c|}{ MS Stars } \\
\hline 122. & $05374+3153$ & 110 & 149 & NO Aur & $\mathbf{M} 2 \mathbf{S}$ & 43 & $\mathrm{E}$ & $6 \%$ & 3331 & Lc & & 0.7 \\
\hline $123 \ldots$ & $07149+0111$ & 217 & 326 & RR Mon & $\mathrm{M} 7 \mathrm{Se}$ & 16 & $\bar{E}$ & 99 & 3332 & $\mathbf{M}$ & 393 & 6.3 \\
\hline $124 \ldots \ldots \ldots$ & $07245+4605$ & 230 & 347 & Y Lyn & M6S & 23 & $\mathrm{E}$ & 25 & 3333 & SRc & 110 & 25.2 \\
\hline $125 \ldots \ldots \ldots$ & $09076+3110$ & 351 & 589 & RS Cnc & M6S & 22 & $\mathrm{E}$ & 11 & 3333 & SRc & 120 & 42.1 \\
\hline $126 \ldots \ldots \ldots$ & $15492+4837$ & 496 & 903 & ST Her & M6.5S & 41 & $\mathrm{E}$ & 53 & 3333 & $\mathrm{SRb}$ & 148 & 49.4 \\
\hline $127 \ldots \ldots \ldots$ & $18076-1034$ & $\ldots$ & $25^{\mathrm{a}}$ & $\ldots$ & M7S & 28 & $\mathrm{E}$ & 99 & 3331 & $\ldots$ & 4.1 & 4.1 \\
\hline $128 \ldots \ldots \ldots$ & $19545-1122$ & $\ldots$ & 1175 & $\ldots$ & M6S & 29 & $\mathrm{E}$ & 99 & 3331 & $\ldots$ & $\ldots$ & 19.6 \\
\hline $129 \ldots \ldots \ldots$ & $00192-2020$ & 7 & 8 & T Cet & M5-6Se & 16 & $\mathrm{~F}$ & 0 & 3333 & $\mathrm{SRb}$ & 159 & 80.2 \\
\hline $130 \ldots \ldots \ldots$ & $07095+6853$ & 210 & 312 & AA Cam & M5S & $\ldots$ & $\mathrm{F}$ & 0 & 3333 & $\mathrm{Lb}$ & $\ldots$ & 27.2 \\
\hline $131 \ldots \ldots \ldots$ & $09411-1820$ & $\ldots$ & 626 & FM Hya & MOS & $\ldots$ & $\mathrm{F}$ & 13 & 3331 & $\mathbf{M}$ & 300 & 25.5 \\
\hline $132 \ldots \ldots \ldots$ & $19226-2012$ & $\ldots$ & 1131 & TT Sgr & M8wkS & $\ldots$ & $\mathrm{F}$ & 99 & 3331 & $\mathbf{M}$ & 333 & 16.3 \\
\hline $133 \ldots \ldots \ldots$ & $23380+7009$ & $\ldots$ & 1339 & $\ldots$ & M6S & $\ldots$ & $\mathrm{F}$ & 33 & 3331 & $\ldots$ & $\ldots$ & 8.4 \\
\hline $134 \ldots \ldots \ldots$ & $04497+1410$ & 84 & 114 & $0^{1}$ Ori & M3S & 18 & $\mathrm{~S}$ & 17 & 3333 & $\mathrm{SRb}$ & 30 & 18.4 \\
\hline $135 \ldots \ldots \ldots$ & $06466-2022$ & 184 & 265 & $\ldots$ & M4S & 16 & $\mathrm{~S}$ & 0 & 3331 & $\ldots$ & $\ldots$ & 9.7 \\
\hline $136 \ldots \ldots \ldots$ & $07392+1419$ & 254 & 382 & NZ Gem & M3S & 18 & $\mathrm{~S}$ & 1 & 3331 & SR & $\ldots$ & 17.5 \\
\hline $137 \ldots \ldots \ldots$ & $16418-1359$ & $\ldots$ & 937 & $\ldots$ & M2S & 19 & $\mathrm{~S}$ & 31 & 3311 & $\ldots$ & $\ldots$ & 20.0 \\
\hline $138 \ldots \ldots \ldots$ & $23070+0824$ & 723 & 1322 & GZ Peg & M4S & 18 & $\mathbf{S}$ & 54 & 3332 & SRa & $\ldots$ & 46.5 \\
\hline $139 \ldots \ldots \ldots$ & $05208-0436$ & $\ldots$ & 134 & V535 Ori & M4wkS & 43 & $\mathrm{U}$ & 21 & 3331 & $\mathrm{Lb}$ & $\ldots$ & 21.7 \\
\hline $140 \ldots \ldots \ldots$ & $18046-0756$ & $\cdots$ & $24^{a}$ & $\ldots$ & M7S & $\ldots$ & I & 72 & 3311 & $\ldots$ & $\ldots$ & 6.1 \\
\hline \multicolumn{13}{|c|}{ SC Stars } \\
\hline $141 \ldots \ldots \ldots$ & $19111+2555$ & 594 & 1112 & S Lyn & $\mathrm{SC}$ & 41 & $\mathrm{E}$ & 99 & 3331 & $\mathbf{M}$ & 438 & 7.1 \\
\hline $142 \ldots \ldots \ldots$ & $13163-6031$ & $\ldots$ & 817 & TT Cen & SC5:/8+ & $\ldots$ & $\mathrm{F}$ & 17 & 3331 & $\mathbf{M}$ & 462 & 1.9 \\
\hline $143 \ldots \ldots \ldots$ & $04491+3825$ & $257^{\mathrm{b}}$ & $797^{\mathrm{c}}$ & $\ldots$ & SC5/9 & 16 & $\mathrm{~S}$ & 15 & 3331 & $\ldots$ & $\ldots$ & 3.5 \\
\hline $144 \ldots \ldots \ldots$ & $04599+1514$ & 87 & 117 & GP Ori & $\mathrm{SC} 7 / 8$ & 17 & $\mathrm{~S}$ & 22 & 3331 & $\mathrm{SRb}$ & 370 & 15.0 \\
\hline $145 \ldots \ldots \ldots$ & $07059+1006$ & $675^{b}$ & $1561^{\mathrm{c}}$ & $\mathrm{R} \mathrm{CMi}$ & $\mathrm{SC} 5 / 10 \mathrm{e}$ & 01 & $\mathbf{S}$ & 98 & 3322 & $\mathbf{M}$ & 338 & 8.3 \\
\hline $146 \ldots \ldots \ldots$ & $12135-5600$ & 442 & 788 & $\ldots$ & $\mathrm{SC}$ & 18 & $\mathbf{S}$ & 46 & 3331 & $\ldots$ & $\ldots$ & 6.3 \\
\hline $147 \ldots \ldots \ldots$ & $13440-5306$ & 470 & 830 & AM Cen & $\mathrm{SC}$ & 18 & $\mathrm{~S}$ & 9 & 3331 & $\mathrm{Lb}$ & $\ldots$ & 8.6 \\
\hline $148 \ldots \ldots \ldots$ & $22036+3315$ & $3107^{b}$ & $5570^{c}$ & RZ Peg & SC6.9, 9 & $\ldots$ & $\mathrm{C}$ & 2 & 3331 & $\mathbf{M}$ & 439 & 17.8 \\
\hline $149 \ldots \ldots \ldots$ & $08461-7051$ & 333 & 556 & $\ldots$ & $\mathrm{SC}$ & $\ldots$ & $\ldots$ & 22 & 3331 & $\ldots$ & $\ldots$ & 17.0 \\
\hline
\end{tabular}

a Star number from Stephenson 1990.

${ }^{b}$ Number in GCGSS.

c Number in GCCGCS.

$08461-7051$ (source 149) has a poor red spectrum, but the blue part looks like a group $\mathrm{S}$ object.

In summary, while there is no clear and definite correlation between the visual photospheric spectral type with the circumstellar classification, a large number of $S$ stars show silicate emission which are very similar to that observed in the circumstellar envelopes of $\mathbf{M}$ stars.

\section{DISTRIBUTION OF S STARS IN THE IRAS COLOR-COLOR DIAGRAM}

Part of the difficulty in deciding the nature of $\mathrm{S}$ stars is that they do not have unique infrared colors and do not form a homogeneous group in the $I R A S$ color-color diagram as in the cases of $M$ and $C$ stars (see $\S 8$ ). $M$ stars on the AGB form a continuous band in the IRAS color-color diagram which can be interpreted as a mass/evolutionary sequence (Olnon et al. 1984; Bedijn 1987; Volk \& Kwok 1988), Visual carbon stars are distributed along a vertical strip near [12] $-[25] \sim 0.2$ (Walker \& Cohen 1988; Chan \& Kwok 1988), whereas infrared carbon stars are distributed along the blackbody line (Chan \& Kwok 1990). However, $S$ stars are not confined to a single region in the $I R A S$ color-color diagram but instead overlap extensively with both regions occupied by $\mathbf{M}$ and $\mathrm{C}$ stars.

In Figure 3 we show the distributions of the $S$ stars in Tables 1 and 2 in the IRAS [25] - [60] versus [12] - [25] color- color diagram. Only objects with good quality fluxes in all three bands are plotted.

We can see that except for a few cases (which will be discussed below), our sample of $\mathbf{S}$ stars is clustered around the blackbody line in the area of $0<[12]-[25]<1.5$ and $-0.5<[25]-[60]<1.5$. It is clear that the group E sources have the reddest colors, and the group $\mathrm{S}, \mathrm{F}$, and $\mathrm{E}$ sources form distinct groups which are progressively distributed toward the direction of decreasing color temperature. The S-F-E color sequence is also consistent with increasing mass-loss rates among these stars.

The three $\mathrm{S}$ stars to show the $\mathrm{SiC}$ feature (group C) are clustered in a small area near the blackbody line at $[12]-[25] \sim 0.6$. Their colors are well within the range of those of infrared carbon stars (Volk, Kwok, \& Langill 1992). The group $F$ sources have bluer colors than the group $C$ objects, and the group $\mathrm{S}$ sources have no color excess $([12]-[25] \sim 0$ and $[25]-[60] \sim 0)$ as expected.

The only source that is out of place (at the upper left-hand corner of the diagram) is source 12 (IRAS $11169-6111$ ), a group E object classified as M 5.5 by Lloyd Evans \& Catchpole (1989). This object has already been noted in $\S 3.2$ to have a peculiar LRS spectrum. From Figure 1 we estimate the 12 to $23 \mu \mathrm{m}$ flux ratio to be $\sim 2$. However, the PSC fluxes for this object are $23.06,4.2$, and $37.38 \mathrm{Jy}$ at 12,25 , and $60 \mu \mathrm{m}$, respec- 

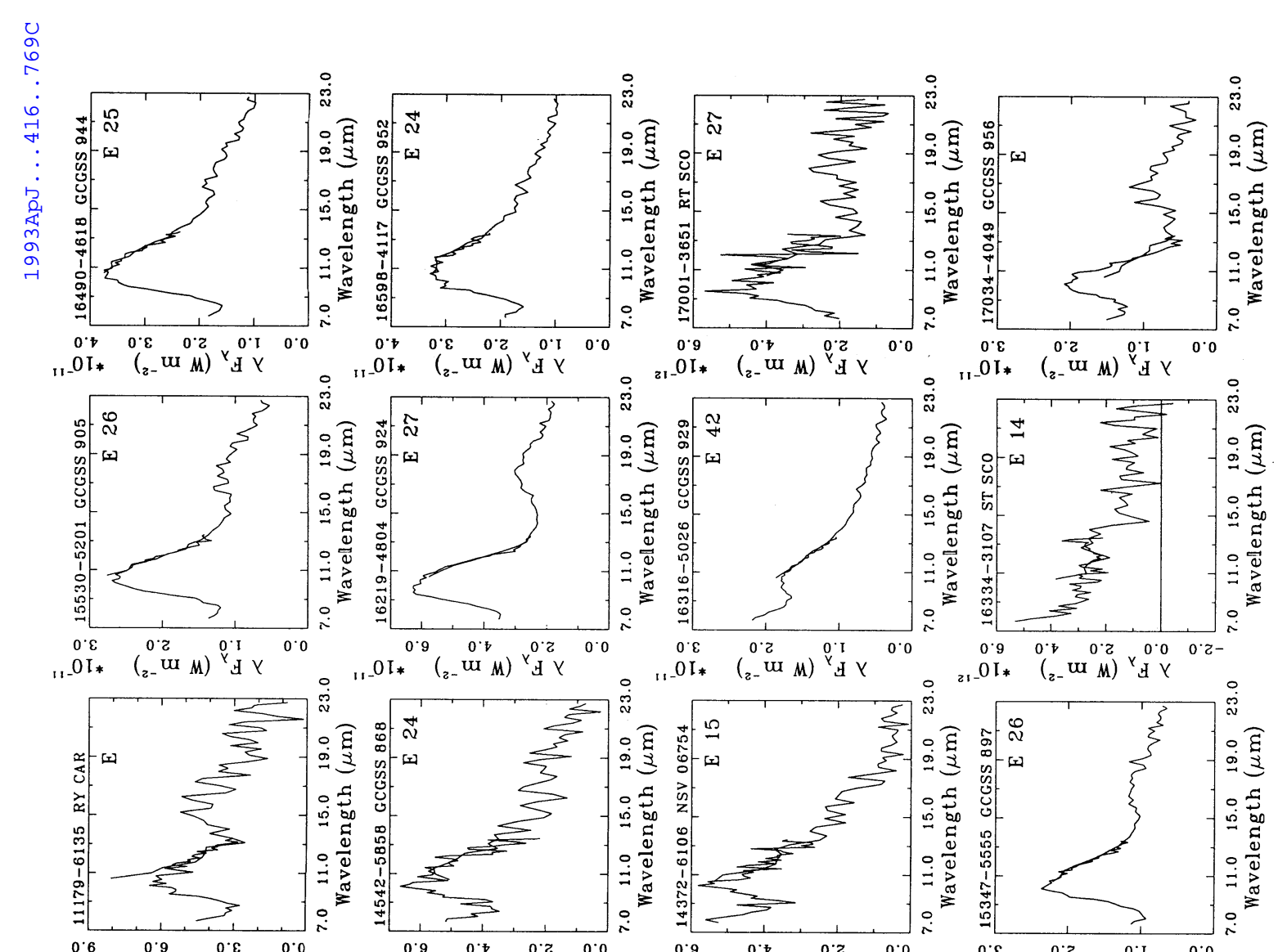

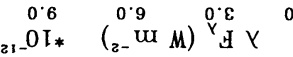
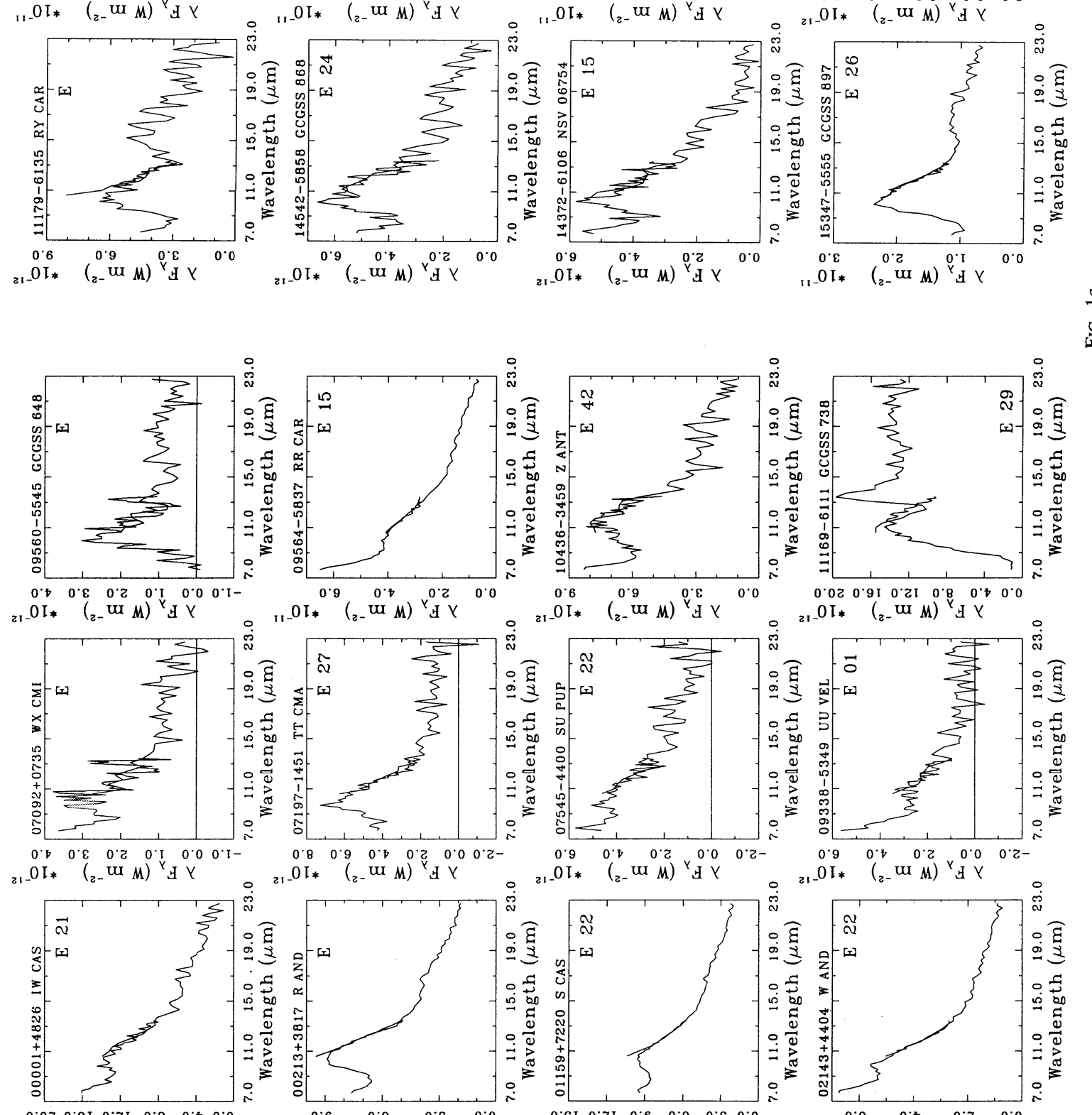

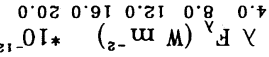
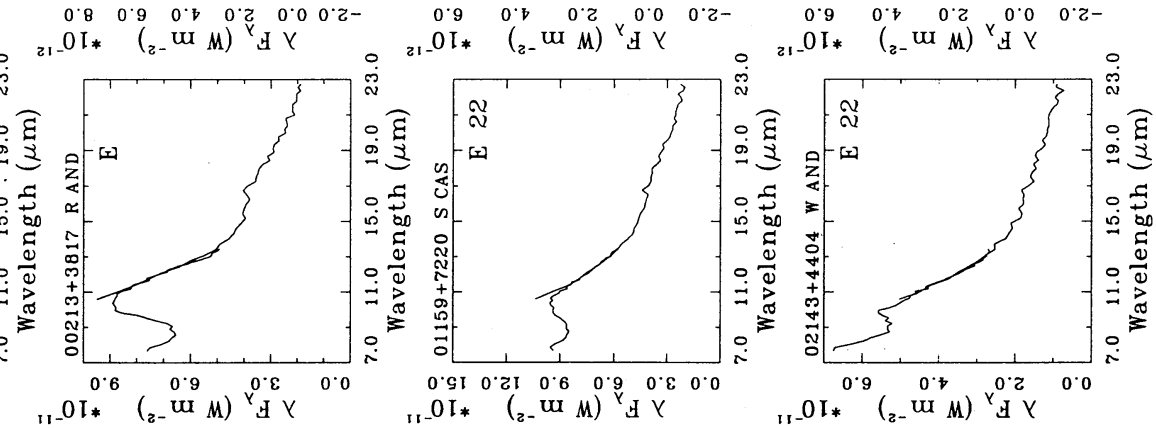

774 

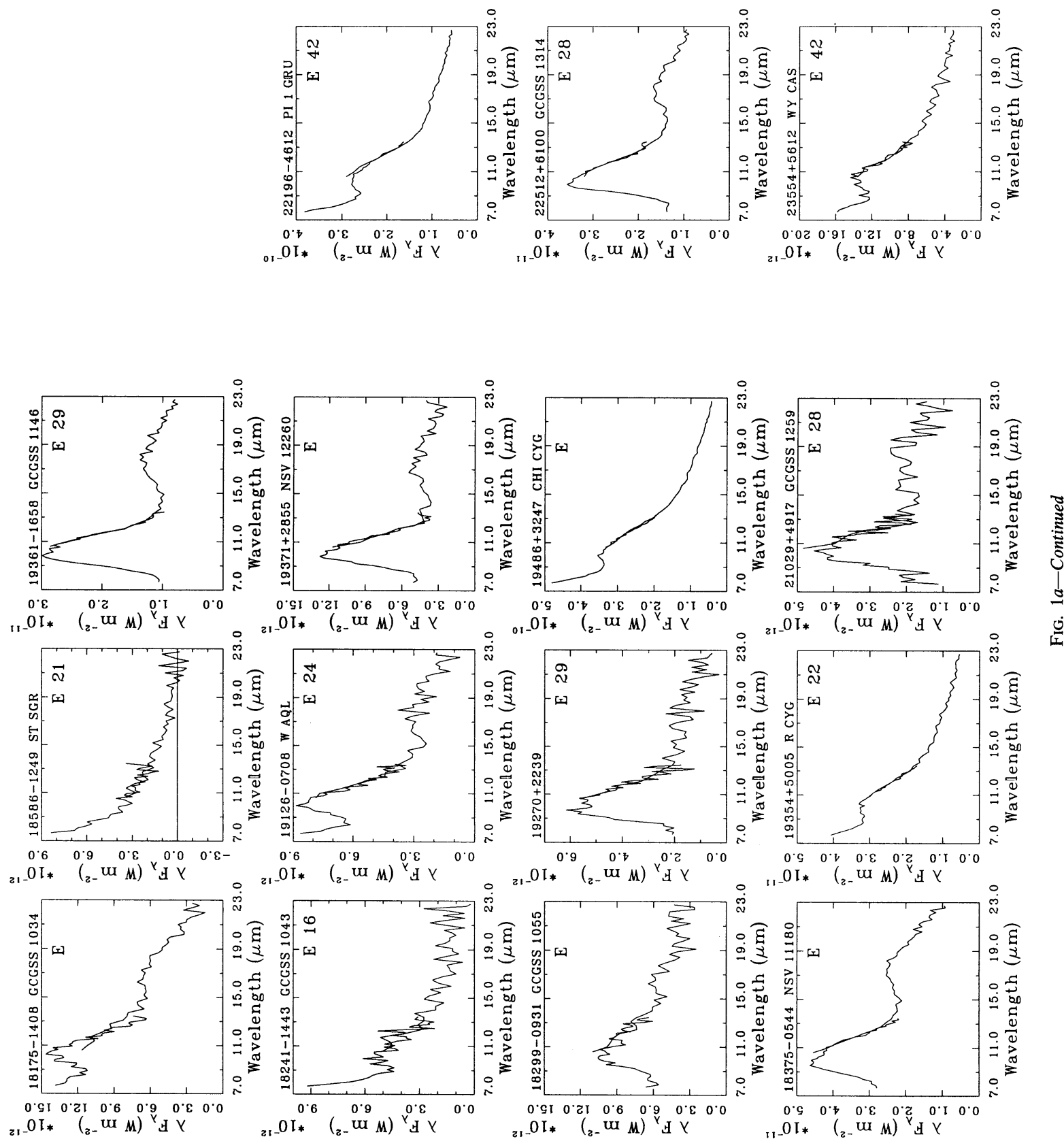

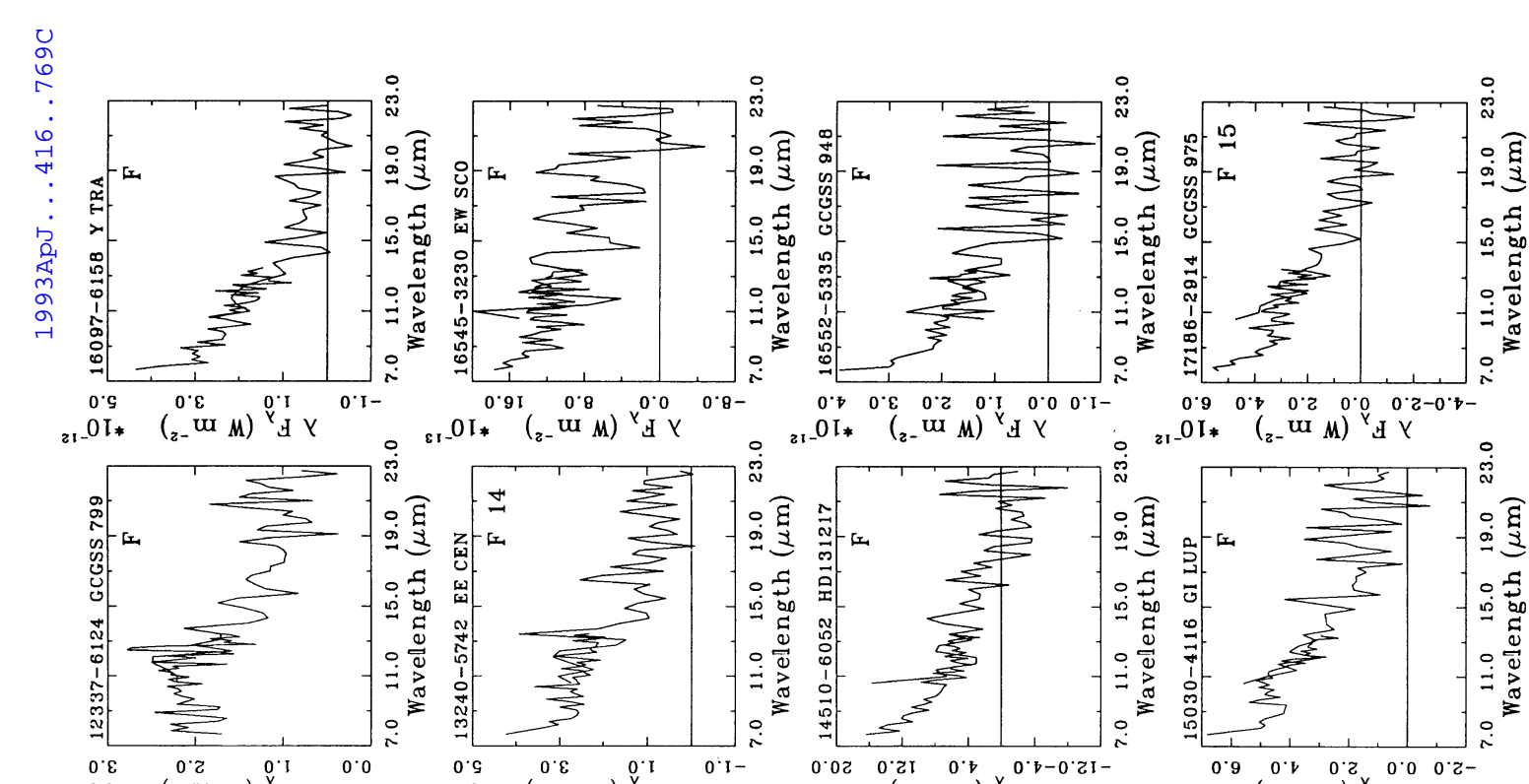

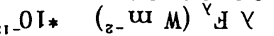
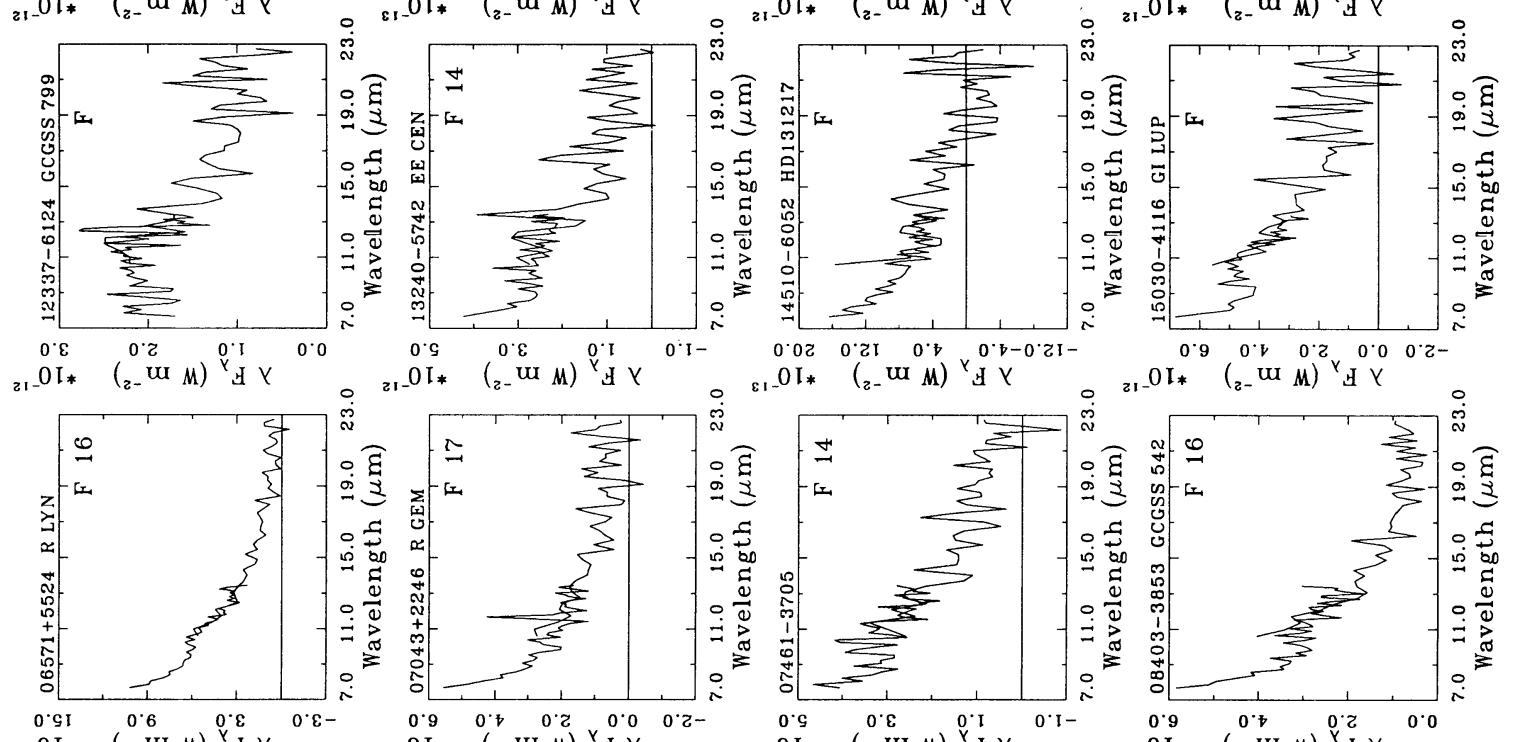

$0.0 z \quad 0.21 \quad 0.7 \quad 0.0-0 . z 1-$
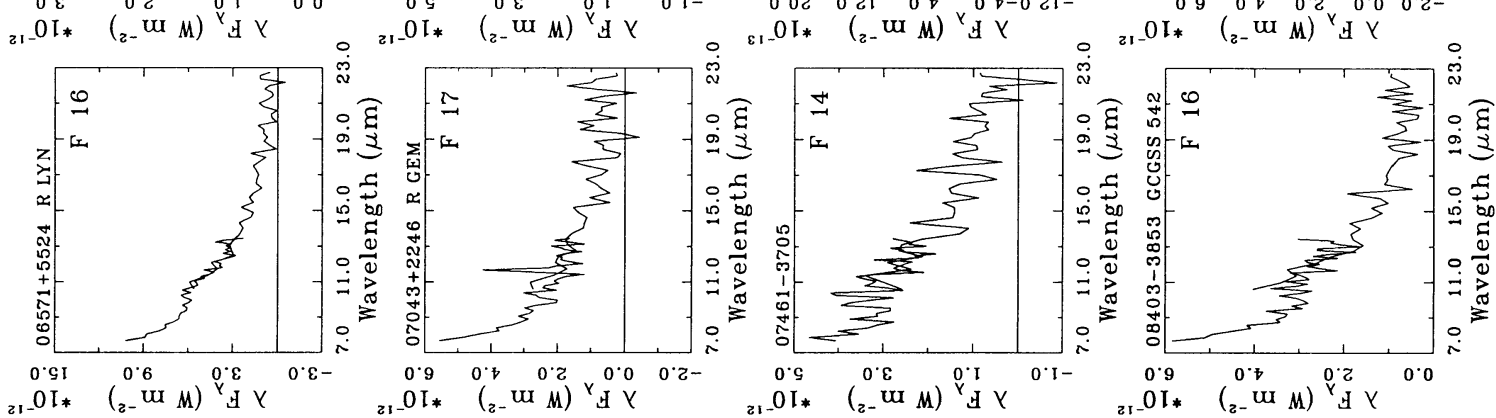

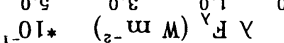

$\stackrel{2}{\dot{9}}$
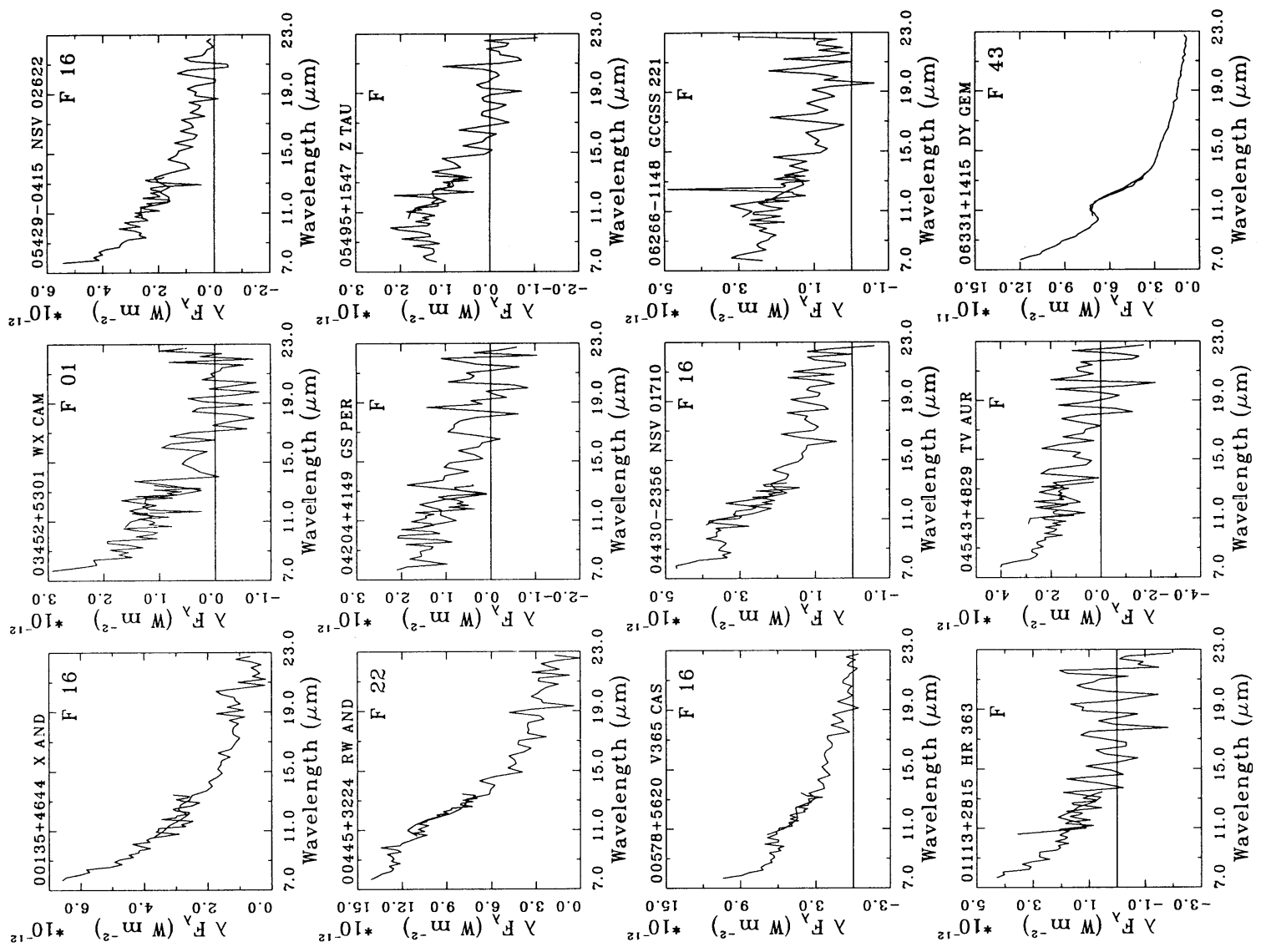

776 

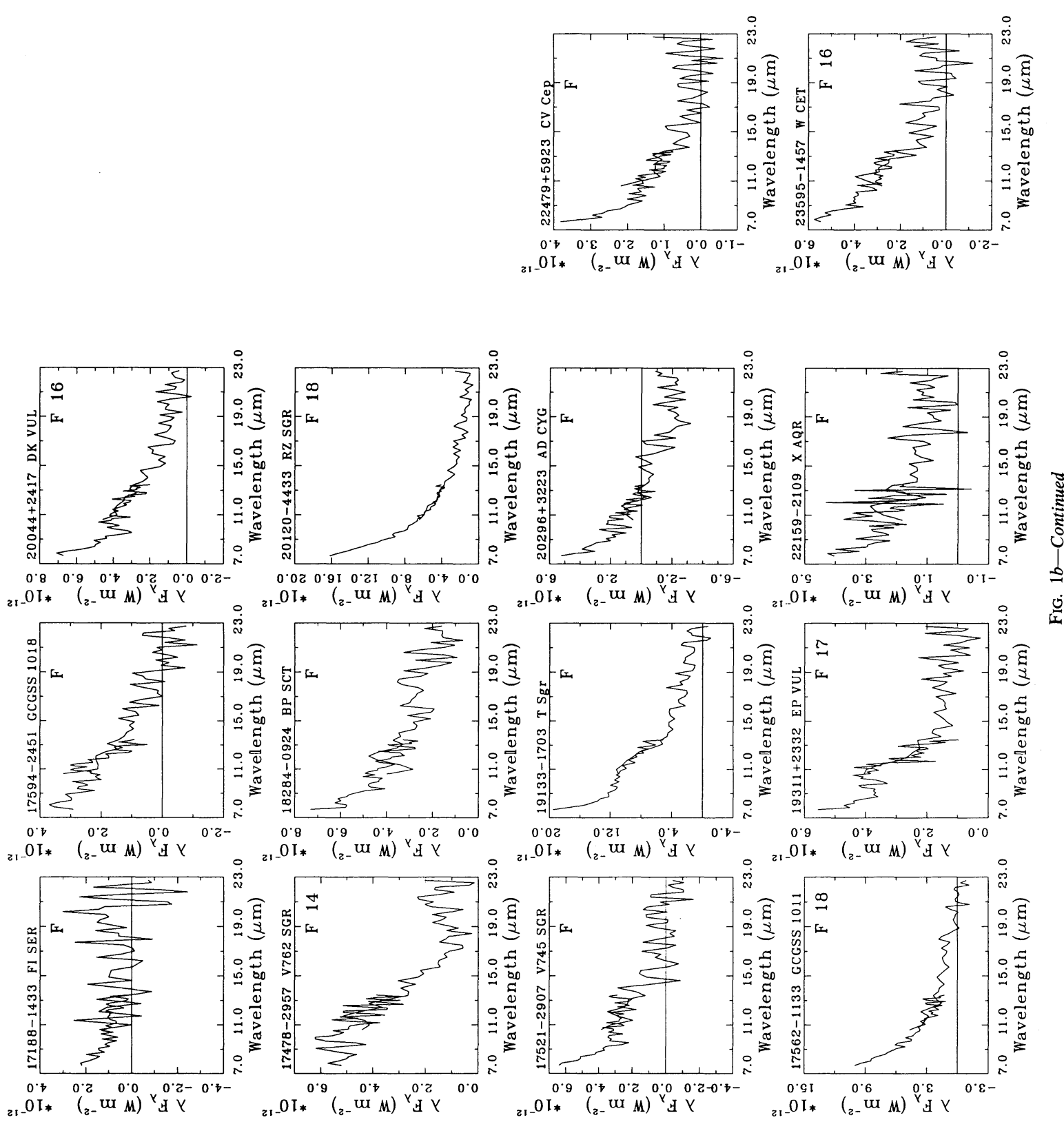

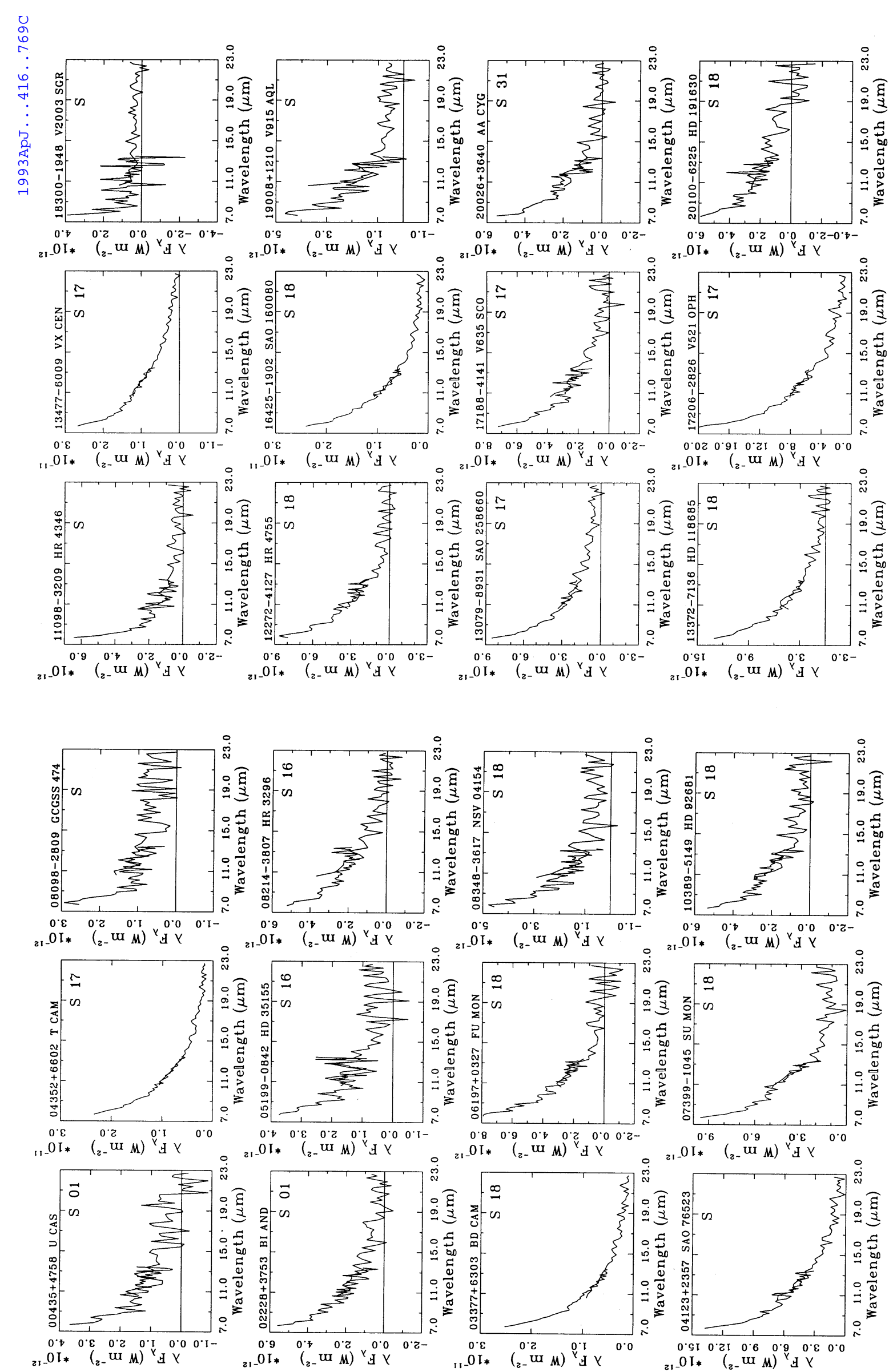

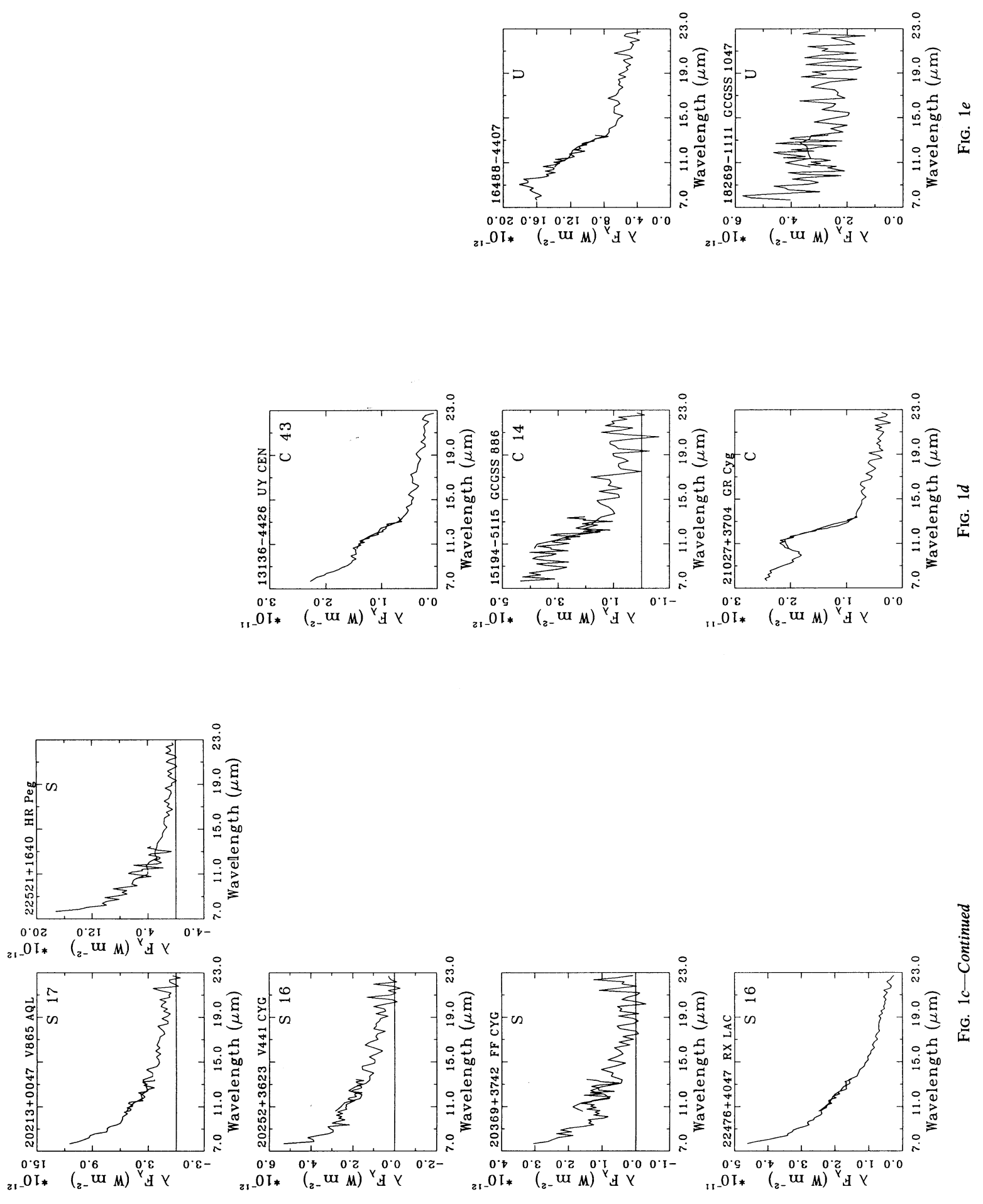

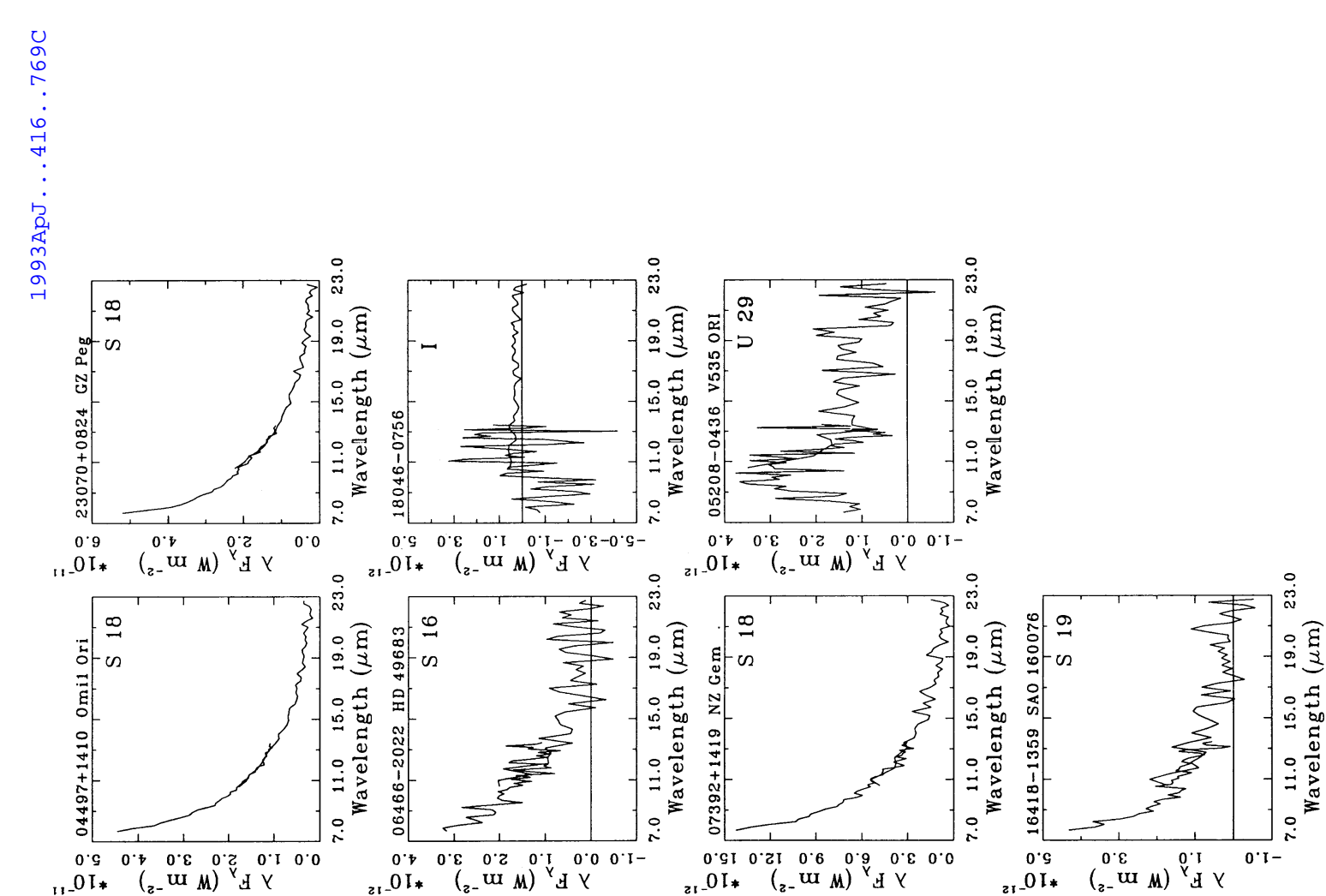

21- $\mathrm{OI}$ *
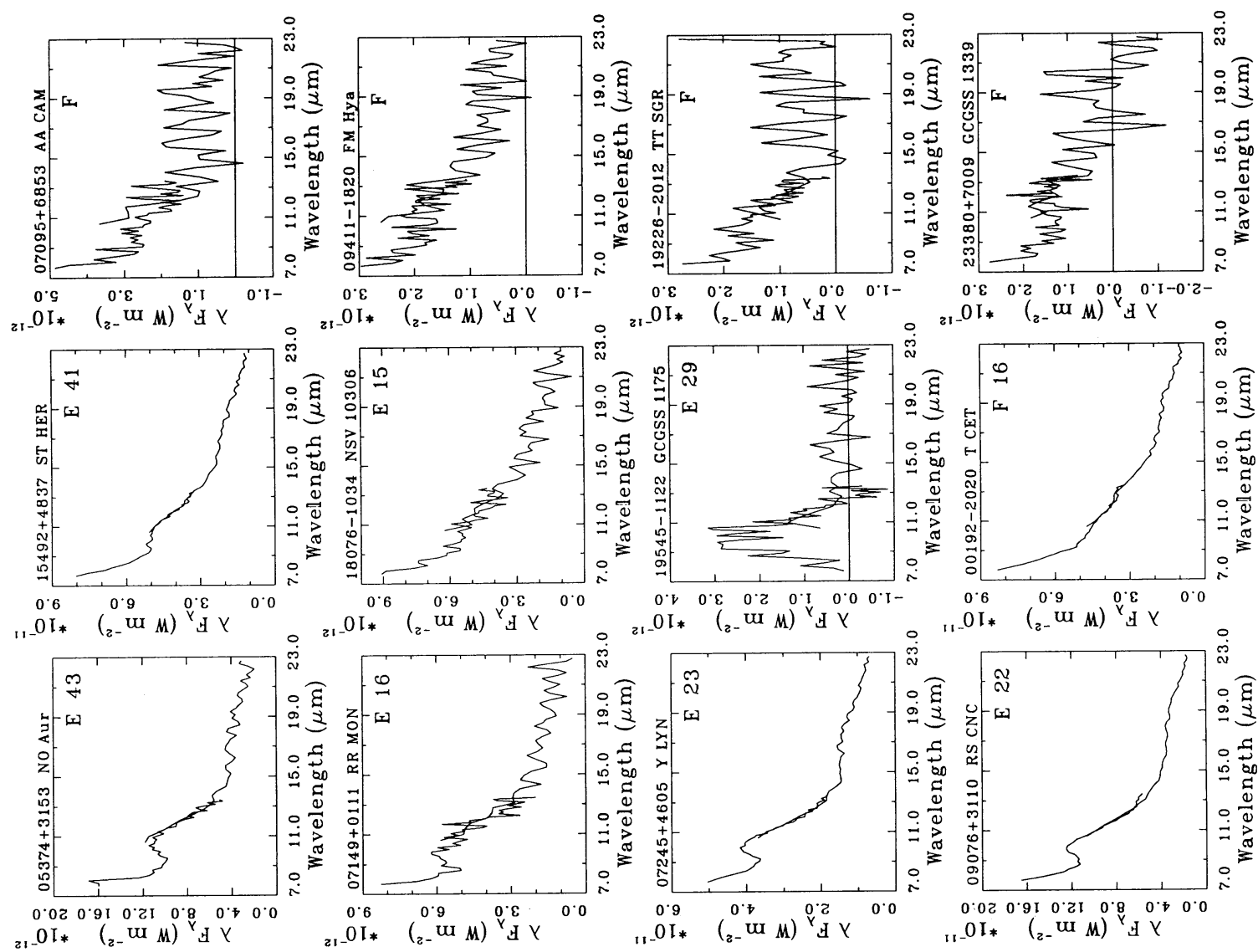

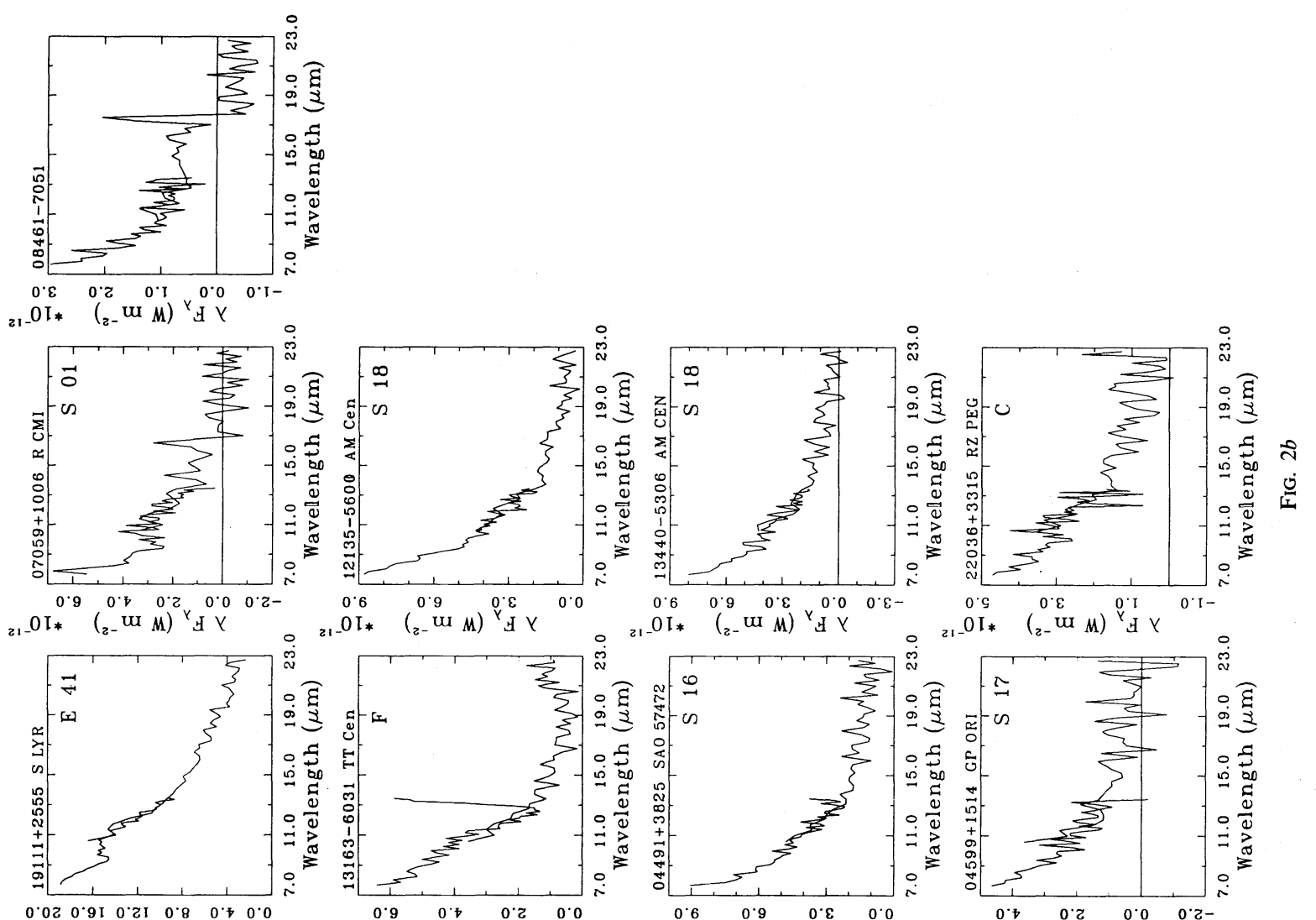

$\begin{array}{llllllllll}0.02 & 0.91 & 0 . z 1 & 0.8 & 0.7 & 0.0\end{array}$

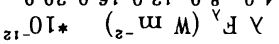
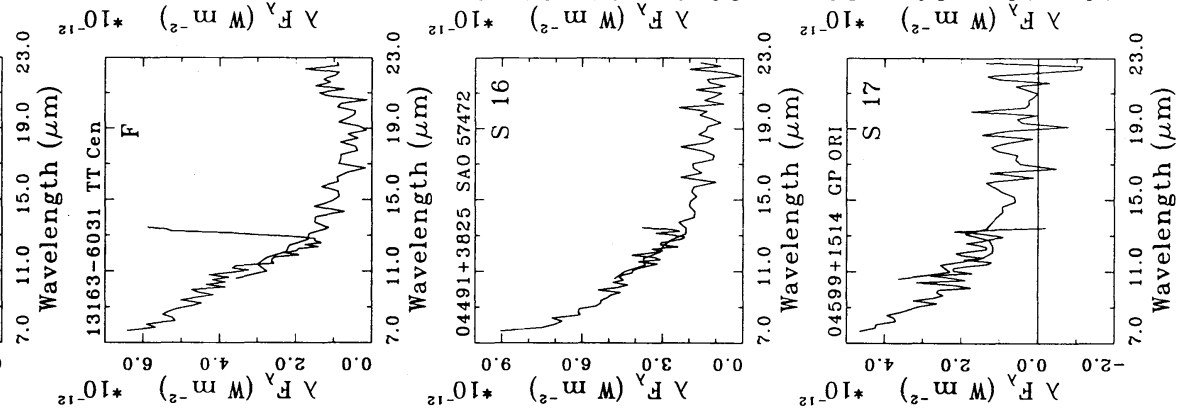


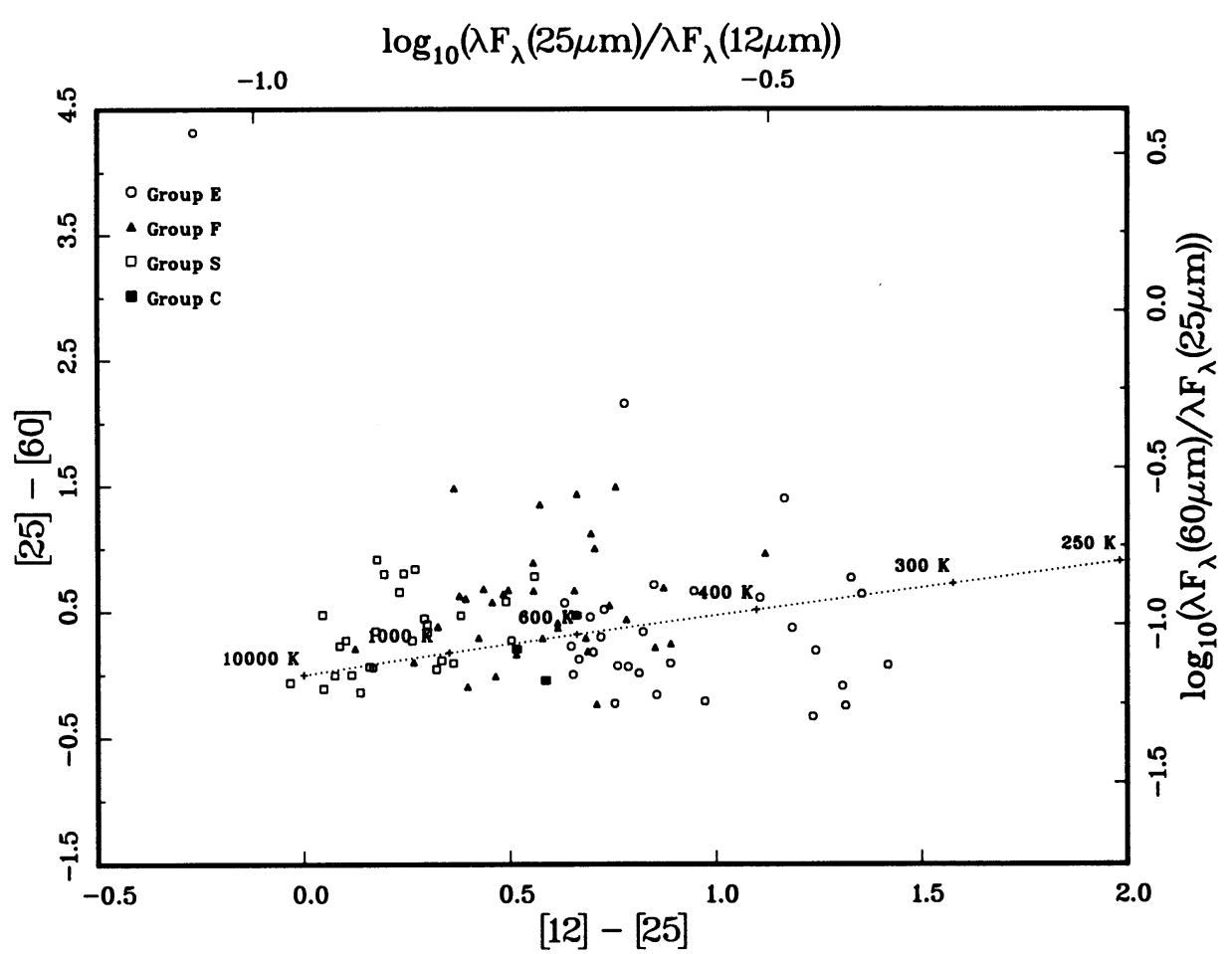

FIG. 3.-The distribution of our $\mathrm{S}$ star sample in the IRAS color-color diagram

tively. It is hard to believe that from 23 to $25 \mu \mathrm{m}$ the flux drops down from above 10 to $4.2 \mathrm{Jy}$. Probably something is wrong with the flux calibration in $25 \mu \mathrm{m}$ for this star. Another star that should be noted is source 35 (IRAS 19486+3247). Although the silicate feature is indeed present in its LRS spectrum (Fig. 1), its colors are more similar to the group $\mathrm{S}$ sources than group $\mathrm{E}$ sources $([12]-[25] \sim 0.15)$.

\section{GALACTIC DISTRIBUTION OF S STARS}

The Galactic distribution of our sample of S stars is plotted in Figure 4. The fact that they are distributed over a wide range of Galactic latitudes implies that they are relatively nearby sources and belong to a local population. However, some differences can be seen among groups E, F, and S. Statistically, the group E sources are slightly closer to the Galactic plane than other groups with $67 \%$ at $|b|<5^{\circ}$ and $80 \%$ at $|b|<10^{\circ}$. In comparison, the group $\mathrm{F}$ sources have $42 \%$ at $|b|<5^{\circ}$ and $63 \%$ at $|b|<10^{\circ}$, and the group $S$ sources have $34 \%$ at $|b|<5^{\circ}$ and $52 \%$ at $|b|<10^{\circ}$. This suggests that the group $\mathrm{S}$ sources are the closest to Earth and the group E sources farthest. This is consistent with the expected selection effect that group $\mathrm{E}$ sources have highest infrared excesses and have a better chance of being detected by IRAS at long wavelengths.

\section{S STARS IN BINARY SYSTEMS}

While most $\mathrm{S}$ stars seem to fit into the M-S-C evolution sequence and their chemical peculiarities with enrichment of $s$-process elements appear to be satisfactorily explained by the

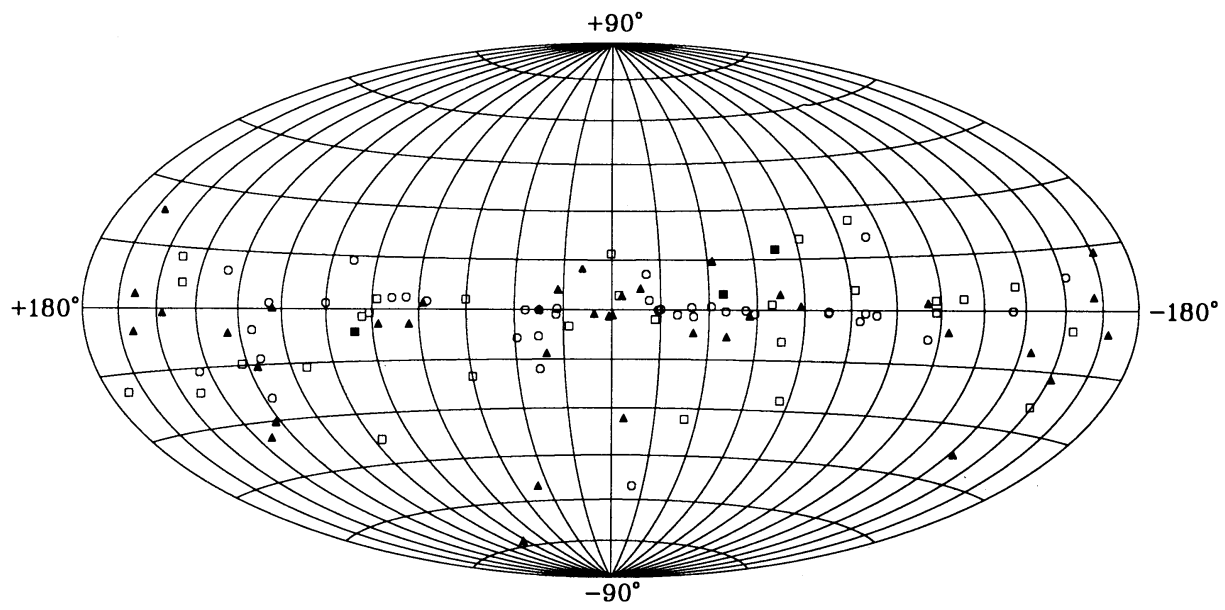

FIG. 4.-The Galactic distribution of S stars. The different symbols represent different LRS groups and are the same as the ones used in Fig. 3. Each grid line corresponds to $15^{\circ}$ in longitude or latitude. 
TABLE 3

Distribution of Tc Presence among the LRS Classes

\begin{tabular}{|c|c|c|c|c|c|c|c|c|}
\hline \multirow[b]{2}{*}{ LRS } & \multicolumn{2}{|r|}{ YES } & \multicolumn{2}{|c|}{ Probable } & \multicolumn{2}{|c|}{ DOUBTFUL } & \multicolumn{2}{|r|}{ No } \\
\hline & $\mathrm{S}$ & MS/SC & $\mathbf{S}$ & MS/SC & $\mathbf{S}$ & MS/SC & $\mathbf{S}$ & $\mathrm{MS} / \mathrm{SC}$ \\
\hline E....... & 5 & 4 & $\ldots$ & $\ldots$ & $\ldots$ & $\ldots$ & $\ldots$ & $\ldots$ \\
\hline$F \ldots \ldots$ & 1 & 1 & $\ldots$ & 1 & $\ldots$ & $\ldots$ & 1 & $\ldots$ \\
\hline S $\ldots \ldots \ldots$ & 5 & 2 & $\ldots$ & $\ldots$ & $\ldots$ & 1 & 2 & 1 \\
\hline $\mathrm{C} \ldots \ldots$ & $\ldots$ & 1 & $\ldots$ & $\ldots$ & $\ldots$ & $\ldots$ & $\ldots$ & $\ldots$ \\
\hline I $\ldots \ldots \ldots$ & 2 & $\ldots$ & $\ldots$ & $\ldots$ & 1 & $\ldots$ & $\ldots$ & $\ldots$ \\
\hline
\end{tabular}

dredge-up theory (cf. Iben \& Renzini 1983), certain number of $\mathrm{S}$ stars stands out by the lack of the unstable element Tc. This has led to the suggestion that the enhanced $s$-process elements found in these stars are the result of mass transfer, and they are not AGB stars at all (Jorissen \& Mayor 1988; Johnson 1992). One of the ways to confirm their AGB nature is through their infrared spectra.

Table 3 shows the distribution of Tc presence among the LRS classes. The Tc data are taken from Little, Little-Marenin, \& Hagen-Bauer (1987) and Smith \& Lambert (1988). We can see that all nine S, MS, and SC stars belonging to group E that have been searched for Tc have Tc present. Among the four stars (HR 363, BD Cam, HD 35155, NZ Gem) with no Tc, one is classified as group $F$ and three others as group $S$. These confirm that the LRS group $E$ is a good indicator of AGB nature of the star, and group $S$ contains objects of doubtful status. We also note that the first three of the four stars with no Tc have been confirmed to be mass-transfer binaries by Johnson (1992). These stars lie very close to the blackbody line in Figure 3 with $[12]-[25] \sim 0.0-0.1$ and $[25]-[60] \sim$ -0.2 to 0.2 , suggesting that they have no or very small infrared excesses (Johnson 1992). We note that the fourth star with no Tc (NZ Gem) is located in same region in the color-color diagram $([12]-[25]=0.11,[25]-[60]=0.02)$ and is therefore quite possible to be in a binary system.

The circumstellar properties of $S$ stars confirm that the $S$ stars that have no Tc in the atmosphere are unlikely to be AGB stars in transition from $\mathbf{M}$ to $\mathrm{C}$ but are first giant branch stars which acquired their $s$-process elements through mass transfer. The $\mathrm{S}$ stars that are in the LRS group $\mathrm{S}$ should be searched for Tc to determine how many of this group are in binary systems.

\section{A NEW CLASSIFICATION SCHEME FOR AGB STARS}

In order to incorporate both the photospheric and circumstellar properties of AGB stars into one classification scheme, we propose the following notation. The spectral class will consist of a main letter and a subscript letter. The main letter refers to the photospheric spectral type $(\mathrm{M}, \mathrm{S}, \mathrm{C})$, and the subscript refers to the circumstellar property (E, A, C, S, F, U). For stars that have subclasses (e.g., M6), the letter code will be placed before the subscript $\left(\mathbf{M} \mathbf{6}_{\mathbf{E}}\right)$.

This scheme will more clearly separate AGB stars in different evolutionary stages. For example, visual carbon stars will be classified as $\mathrm{C}_{\mathrm{S}}$ (cf. Chan \& Kwok 1988), infrared carbon stars as $C_{C}$ (cf. Chan \& Kwok 1990), extreme carbon stars as $\mathrm{C}_{\mathrm{U}}$ (cf. Volk et al. 1992), and carbon stars with silicate features as $C_{E}$ (cf. Little-Marenin 1986). Similarly, $M$ stars will be classified as $\mathbf{M}_{\mathbf{S}}, \mathbf{M}_{\mathrm{F}}, \mathbf{M}_{\mathbf{E}}$ depending on the degree of mass loss, and those with very high mass-loss rates (e.g., some OH/IR stars) will be classified as $\mathbf{M}_{\mathrm{A}}$, although in practice only the circumstellar spectra are available.

\section{THE EVOLUTIONARY STATUS OF S STARS}

From the LRS spectra of a sample of $149 \mathrm{~S}$ stars, we find that their circumstellar properties can be divided into three main groups: (1) stars with large infrared excesses which show the silicate emission feature $\left(\mathrm{S}_{\mathrm{E}}\right.$ stars $) ;(2)$ stars with modest amount of circumstellar dust ( $\mathrm{S}_{\mathrm{F}}$ stars); and (3) stars with no infrared excess $\left(S_{S}\right.$ stars). A small number of $S$ stars show the $11.3 \mu \mathrm{m} \mathrm{SiC}$ feature commonly found in carbon stars $\left(\mathrm{S}_{\mathrm{C}} \mathrm{stars}\right)$. There is no clear one-to-one relationship between the optical spectral types and the circumstellar properties except that the S-type Mira variables generally have larger infrared excesses.

The most striking feature of the circumstellar spectra of $S$ stars in Figure 1 is that they are indistinguishable from the circumstellar spectra of $M$ stars. It suggests that the circumstellar envelopes of S stars may in fact represent the circumstellar envelopes of $\mathrm{M}$ stars, and only the photospheric composition of the stars has changed. The S-F-E color sequence in the IRAS color-color diagram is not an evolutionary sequence but is instead the result of separate paths of evolution from $\mathbf{M}$ stars. Specifically, $S_{S}$ stars evolve from $M$ stars with no mass loss ( $M_{S}$, probably of low mass), $S_{F}$ stars evolve from $M$ stars with low mass-loss rates $\left(M_{F}\right)$, and $S_{E}$ stars evolve from $M$ stars with significant rates of mass loss ( $\mathbf{M}_{E}$, probably stars of high mass). As long as there is adequate production of silicate grains, $S_{E}$ stars will be still losing mass. Mass loss will terminate when the supply of oxygen is exhausted as they are all tied up in $\mathrm{CO}$ when $[\mathrm{C} / \mathrm{O}]$ approaches unity. The transition from $\mathrm{M}$ to $\mathrm{S}$ stars does not cause any major movement in the IRAS colorcolor diagram.

In this picture, the carbon stars with silicate features $\left(C_{E}\right.$ stars) do not descend directly from $M$ stars, but instead descend from $S_{E}$ stars. Figure 5 shows the distributions of the $\mathrm{S}_{\mathrm{E}}$ stars and $\mathrm{C}_{\mathrm{E}}$ stars in the IRAS color-color diagram. To this date, there are 15 carbon stars found to have silicate features. These include seven from Little-Marenin (1986) and Willems \& de Jong (1986) after eliminating 13 misclassified candidates (Lloyd Evans 1990), four from Chan \& Kwok (1991), and four from Chan, Kwok, \& Volk (1992) and LeVan et al. (1992). Twelve of the 15 have three good IRAS colors, and they are plotted in Figure 5. When we compare the colors of these stars to the evolutionary tracks of Chan \& Kwok (1988), we can see $\mathrm{S}_{\mathrm{E}} \rightarrow \mathrm{C}_{\mathrm{E}}$ hypothesis fits into the general evolutionary scenario of Kwok \& Chan (1990), where $C_{E}$ stars will evolve to visual carbon stars with far-infrared excesses and the to carbon stars with $\mathrm{SiC}$ features (hereafter $\mathrm{C}_{\mathrm{C}}$ stars).

What about $\mathrm{S}$ stars with $\mathrm{SiC}$ feature $\left(\mathrm{S}_{\mathrm{C}}\right.$ stars $)$ ? It is possible that for more massive $M$ stars, transition to $S$ stars is so rapid that the large increase in the photospheric abundance of carbon allows an early condensation of SiC. These stars will not go through the visual carbon star stage, but evolve directly from $S$ stars to infrared carbon stars.

\section{EVOLUTION ON THE ASYMPTOTIC GIANT BRANCH}

The change in surface chemical abundance of AGB stars is a manifestation of the internal changes as a star evolves. Such changes in chemical composition also lead to changes in the mass loss process, resulting in different circumstellar properties. While the main sequence in the Hertzsprung-Russell (H-R) diagram is primarily a mass sequence, the AGB is a superposition of mass and evolutionary sequences. The $H-R$ diagram is not the best tool to study the M-S-C evolution for $\mathbf{M}, \mathrm{S}$, and $\mathrm{C}$ stars have similar photometric properties 


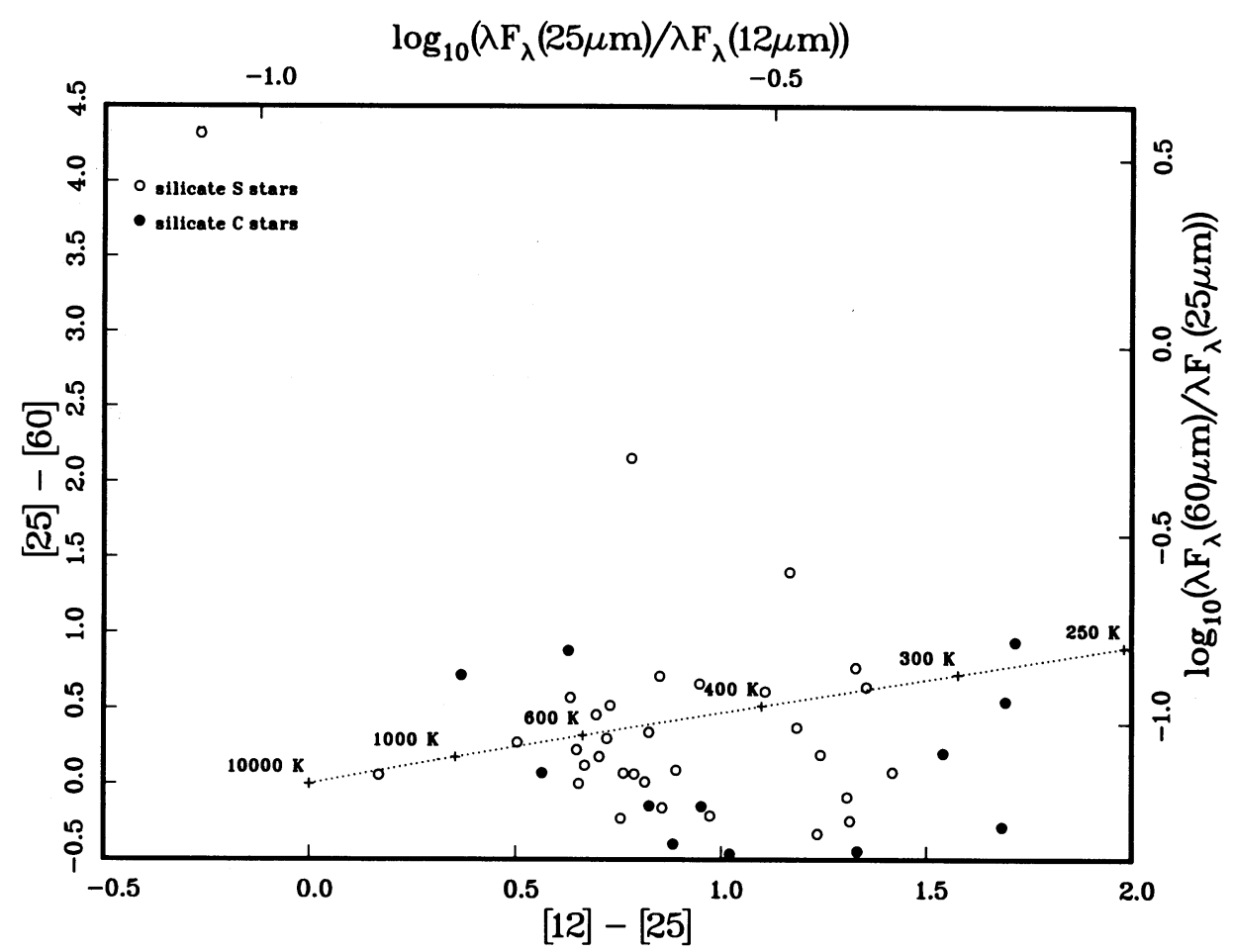

FIG. 5.-The distribution of $\mathrm{S}$ and $\mathrm{C}$ stars with silicate emission features in the $I R A S$ color-color diagram

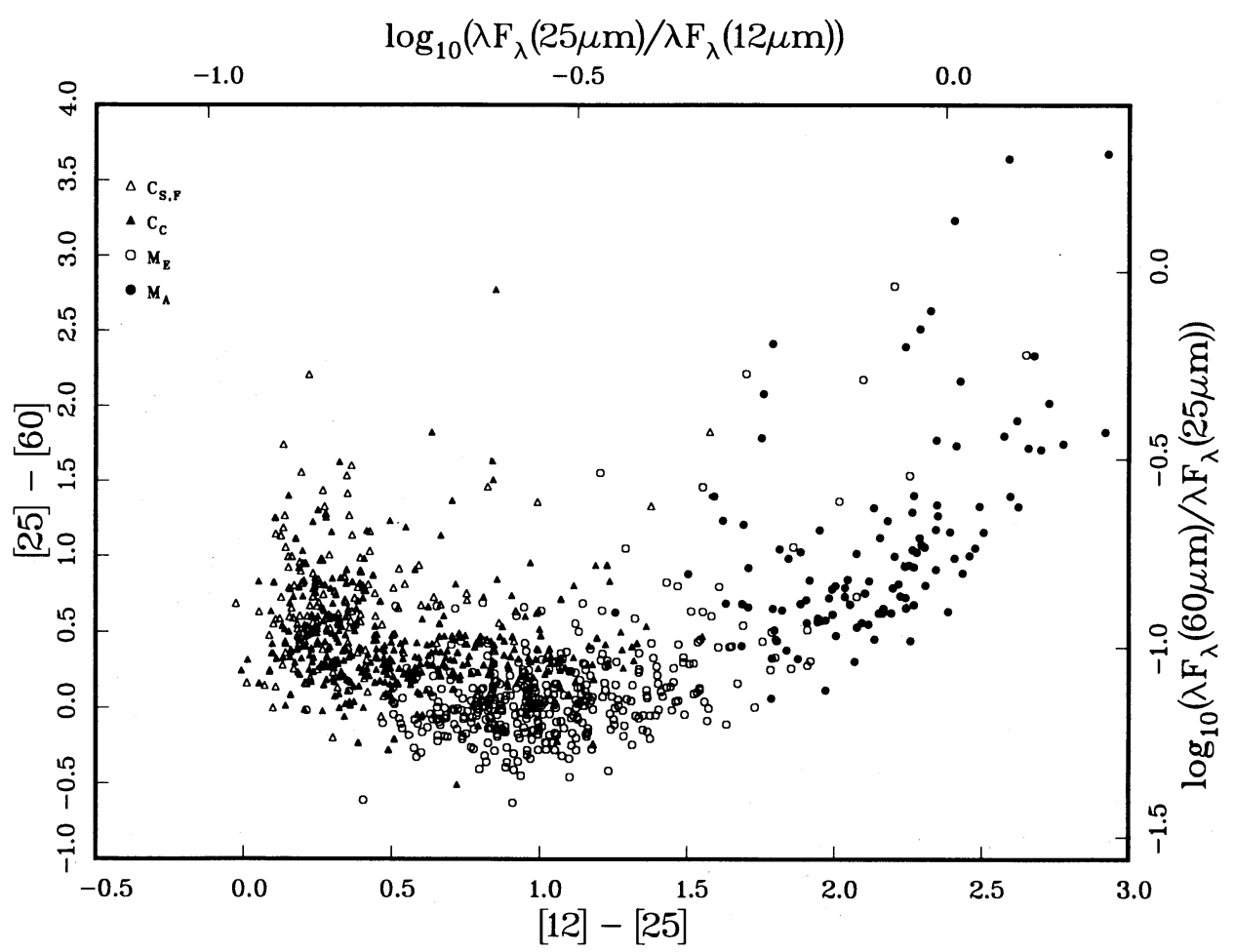

FIG. 6. - The distribution of oxygen- and carbon-rich stars in the IRAS color-color diagram. The number of stars plotted are 399, 160, 395, and 114 for $\mathrm{C}_{\mathrm{C}}, \mathrm{C}_{\mathrm{S}}$ $\mathrm{M}_{\mathrm{E}}$, and $\mathrm{M}_{\mathrm{A}}$ stars, respectively. Because of the large numbers of $\mathrm{M}_{\mathrm{E}}$ stars, only those with four good $I R A S$ colors are plotted. 
(luminosity and temperature), and they all lie on the same tracks. The IRAS color-color diagram is better suited for this purpose. Figure 6 shows the distribution of $M_{E}, M_{A}, C_{S}+C_{F}$, and $\mathrm{C}_{\mathrm{C}}$ stars in the IRAS color-color diagram. The $\mathrm{C}_{\mathrm{S}}+\mathrm{C}_{\mathrm{F}}$ group contains 160 carbon stars in GCCGCS which LRS spectra are classified as group $\mathrm{S}$ or $\mathrm{F}$, and the $\mathrm{C}_{\mathrm{C}}$ group contains 399 GCCGCS stars that are classified as group $\mathrm{C}$. The $\mathrm{M}_{\mathrm{E}}$ and $\mathrm{M}_{\mathrm{A}}$ groups are IRAS LRS sources that belong to groups $\mathrm{E}$ and $\mathrm{A}$, respectively. We can see that there are two separate sequences: the oxygen sequence $\mathrm{M}_{\mathrm{E}} \rightarrow \mathrm{M}_{\mathrm{A}}$ (shown as open and filled circles) and the carbon sequence $\mathrm{C}_{\mathrm{S}, \mathrm{F}} \rightarrow \mathrm{C}_{\mathrm{C}}$ (shown as open and filled triangles). The $\mathrm{C}_{\mathrm{S}, \mathrm{F}}$ stars are distributed along a vertical strip near [12] $-[25] \sim 0.2$ and then turn horizontal to meet the $\mathrm{C}_{\mathrm{C}}$ stars which extend to $[12]-[25] \sim 1.5$. The oxygen sequence starts as $[12]-[25] \sim 0.5$ and curve up to the upper right. Over the region where the [12] - [25] colors of the two sequences overlap, they are separated in the [25] - [60] colors (the carbon sequence having larger [25] - [60] indices). Presumably, the AGB is terminated at the ends of either $C_{C}$ or $M_{A}$ (Kwok \& Chan 1990). The evolutionary connection between the two sequences is less clear, although the hypothesis of Willems \& de Jong (1988) of a fast evolutionary loop from $\mathbf{M}_{\mathbf{E}}$ to $C_{S}$ is possible (Chan \& Kwok 1988).

We should note that a complete interpretation of Figure 6 will require not only the evolutionary tracks but also a superposition of tracks for different masses. For example, probably only the most massive oxygen-rich stars go to the reddest colors (Volk \& Kwok 1988). Our inability to separate the mass and evolutionary effects remains a major obstacle to the understanding of AGB evolution.

\section{CONCLUSIONS}

Stars on the asymptotic giant branch can be classified according to their photospheric and circumstellar spectra. In this paper, we have made an attempt to reconcile these two separate classifications, particularly for S stars which are commonly believed to be transition objects between $M$ and $C$ stars. We use the notation of two letters (e.g., $S_{E}$ ), where the main letter refers to the photospheric spectral type and the subscript refers to the circumstellar classification. This notation has the advantage of separating stars which have similar photospheric compositions but which are in different stages of AGB evolution. This notation also recognizes the importance of mass loss on the evolution on the AGB.

We note that part of the reason for $\mathrm{S}$ stars not having homogeneous mid-infrared colors is due to the contamination by mass transfer. While only four cases have been identified through the search of Tc, a more complete survey is needed to exclude these stars so that a sample of true transition objects can be assembled. We suggest that $S_{S}$ stars are the most likely candidates for the search.

We propose the following scenario for the evolution of $\mathrm{M}, \mathrm{S}$, and $\mathrm{C}$ stars on the AGB. Stars start on the AGB first as M stars. As the result of dredge-up, the [C/O] ratio in the photosphere steadily increases. Depending on the initial mass of the star, the transition to $S$ stars will occur when the star has no mass loss $\left(\mathrm{M}_{\mathrm{S}}\right)$, little mass loss $\left(\mathrm{M}_{\mathrm{F}}\right)$, or significant mass loss $\left(\mathrm{M}_{\mathrm{E}}\right)$. However, since the $[\mathrm{C} / \mathrm{O}]$ ratio remains under unity, the production of silicate grains is not affected and mass loss (if already present) continues as before. When enough carbon is dredged up, the photospheric classification of the star changes from S to C, and mass loss is interrupted. This leads to the creation of carbon stars with silicate features. The following schematic sequence illustrates the evolution:

For stars with low initial masses:

$$
\mathrm{M}_{\mathrm{s}} \rightarrow \mathrm{S}_{\mathrm{S}} \rightarrow \mathrm{C}_{\mathrm{s}}
$$

For stars with higher initial masses:

$$
\mathrm{M}_{\mathrm{F}} \rightarrow \mathrm{S}_{\mathrm{F}} \rightarrow \mathrm{C}_{\mathrm{F}}
$$

For stars with even higher initial masses:

$$
\begin{aligned}
\mathrm{M}_{\mathrm{E}} \rightarrow \mathrm{S}_{\mathrm{E}} \rightarrow \mathrm{C}_{\mathrm{E}} & \rightarrow \text { visual carbon stars }\left(\mathrm{C}_{\mathrm{S}}\right) \\
& \rightarrow \text { infrared carbon stars }\left(\mathrm{C}_{\mathrm{C}}\right) \\
& \rightarrow \text { extreme carbon stars }\left(\mathrm{C}_{\mathrm{U}}\right)
\end{aligned}
$$

For stars with very high initial masses:

$$
M_{E} \rightarrow M \text { stars with silicate feature in absorption }\left(M_{A}\right)
$$

This scenario reconciles the photospheric evolutionary sequence of $M \rightarrow S \rightarrow C$, and the circumstellar properties of $M$, $S$, visual carbon, and infrared carbon stars. We note, however, the step from $C_{E}$ to $C_{S}$ is the most uncertain one for the lack of transition objects. The evidence of this step is primarily based on the common occurrence of $60 \mu \mathrm{m}$ excesses observed in $\mathrm{C}_{\mathrm{S}}$ stars.

Finally, we suggest that the circumstellar spectra of AGB stars provide important supplementary information on the evolutionary status of AGB stars and should be treated as equal to the photospheric spectra in the classification of the stars.

We thank William Bidelman and Kevin Volk for helpful discussions, and Paul Wesselius for providing the Groningen LRS database. P. S. C. acknowledges the support of the C. C. Huang Foundation of the Academia Sinica. This work is supported by a grant to SK from the Natural Sciences and Engineering Research Council of Canada.

\section{REFERENCES}

Atlas of Low Resolution IRAS Spectra. 1986, IRAS Science Team, prepared by F. M. Olnon \& E. Raimond (A\&AS, 65, 607) (LRS Atlas)

Bedijn, P. J. 1987, A\&A, 186, 136

Chan, S. J., \& Kwok, S. 1988, ApJ, 334, 362

$$
\text { 1990, A\&A, 237, } 354
$$

Chen, P. S., Gao, H., Chen, Y. K., \& Dong, H. W. 1988 A\&AS, 72, 239

HST Guide Star Catalog. 1989 (Baltimore: STScI) (Astronomical Data Center CD-ROM Selected Astronomical Catalog, 1)

IRAS Point Source Catalog, Version 2. 1988, Joint IRAS Science Working Group (Washington, DC: GPO) (PSC)
Johnson, H. R. 1992, in Evolution Processes in Interacting Binary Stars, ed. Y. Kondo et al. (Dordrecht: Kluwer), 157

Jorissen, A., \& Mayor, M. 1988, A\&A, 198, 187

Keenan, P. C., \& Boeshaar, P. C. 1980, ApJS, 43, 379

Kholopov, P. N., ed. 1985, General Catalogue of Variable Stars (4th ed.; Moscow: Nauka) (GCVS)

Kwok, S., \& Chan, S. J. 1990, in From Miras to Planetary Nebulae: Which Path of Evolution?, ed. M. O. Mennessier \& A. Omont (Gif-sur-Yvette: Editions Frontières), 297 $\underset{373}{\text { Lambert, D. L., Gustafsson, B., Eriksson, K., \& Hinkle, K. H. 1986, ApJS, 62, }}$ 
LeVan, P. D., Sloan, G. C., Little-Marenin, I. R., \& Grasdalen, G. L. 1992, ApJ, 392,702

Little, S. J., Little-Marenin, I. R., \& Hagen-Bauer, W. 1987, AJ, 94, 981

Little-Marenin, I. R. 1986, ApJ, 307, L15

Little-Marenin, I. R., \& Little, S. J. 1988, ApJ, 333, 305

' Ll Lloyd Evans, T. 1990, MNRAS, 243, 336

I L Lloyd Evans, T., \& Catchpole, R. M. 1989, MNRAS, 237, 219

- Noguchi, K., Sun, J. H., \& Wang, G. 1991, PASJ, 43, 311

Olnon, F. M., Baud, B., Habing, H. J., de Jong, T., Harris, S., \& Pottasch, S. R. 1984, ApJ, 278, L1

Stephenson, C. B. 1976, Pub. Warner \& Swasey Obs., No. 2 (GCSS) . 1984, Pub. Warner \& Swasey Obs., 3, No. 1 (GCGSS)

Stephenson, C. B. 1989, Pub. Warner \& Swasey Obs., 3, No. 2 (GCCGCS) -1990, AJ, 100, 569
Skinner, C. J., Griffin, I., \& Whitmore, B. 1990, MNRAS, 243, 78

Smith, V. V., \& Lambert, D. L. 1985, ApJ, 294, 326 1986, ApJ, 311, 843

$1988, \mathrm{ApJ}, 333,219$

Treffers, R., \& Cohen, M. 1974, ApJ, 188, 545

van der Veen, W. E. C. J., \& Habing, H. 1988, A\&A, 194, 125

Volk, K. 1993, in Astronomical Infrared Spectroscopy: Future Observational

Directions, ed. S. Kwok (ASP Conf. Ser., 41), 63

Volk, K., \& Cohen, M. 1989, AJ, 98, 931

Volk, K., \& Kwok, S. 1988, ApJ, 331, 435

Volk, K., Kwok, S., \& Langill, P. P. 1992, ApJ, 391, 285

Volk, K., Kwok, S., Stencel, R. E., \& Brugel, E. 1991, ApJS, 77, 607

Walker, H., \& Cohen, M. 1988, AJ, 95, 1801

Willems, F. J., \& de Jong, T. 1988, A\&A, 196, 173

Woolf, N. J., \& Ney, E. P. 1969, ApJ, 155, L181 